\title{
Canonical form of three-fermion pure-states with six single particle states
}

\author{
Lin Chen ${ }^{1,2,3}$, Dragomir Ž Đoković ${ }^{1,2}$, Markus Grassl ${ }^{3}$, Bei Zeng ${ }^{2,4}$ \\ ${ }^{1}$ Department of Pure Mathematics, University of Waterloo, Waterloo, Ontario, Canada \\ ${ }^{2}$ Institute for Quantum Computing, University of Waterloo, Waterloo, Ontario, Canada \\ ${ }^{3}$ Centre for Quantum Technologies, National University of Singapore, Singapore \\ ${ }^{4}$ Department of Mathematics \& Statistics, University of Guelph, Guelph, Ontario, Canada
}

June 6, 2013

\begin{abstract}
We construct a canonical form for pure states in $\wedge^{3}\left(\mathbb{C}^{6}\right)$, the three-fermion system with six single particle states, under local unitary (LU) transformations, i.e., the unitary group $\mathrm{U}(6)$. We also construct a minimal set of generators of the algebra of polynomial $\mathrm{U}(6)$-invariants on $\wedge^{3}\left(\mathbb{C}^{6}\right)$. It turns out that this algebra is isomorphic to the algebra of polynomial LU-invariants of three-qubits which are additionally invariant under qubit permutations. As a consequence of this surprising fact, we deduce that there is a one-to-one correspondence between the $\mathrm{U}(6)$-orbits of pure three-fermion states in $\wedge^{3}\left(\mathbb{C}^{6}\right)$ and the LU orbits of pure three-qubit states when qubit permutations are allowed. As an important byproduct, we obtain a new canonical form for pure three-qubit states under LU transformations $\mathrm{U}(2) \times \mathrm{U}(2) \times \mathrm{U}(2)$ (no qubit permutations allowed).
\end{abstract}

\section{Contents}

1 Introduction $\quad 2$

2 Related Work

2.1 Systems of distinguishable particles . . . . . . . . . . . . . 5

2.2 Fermionic systems . . . . . . . . . . . . . . . . . . . . . . . . . . . . . . . . . . . . . . . .

2.3 Geometric measure of entanglement . . . . . . . . . . . . . . . 7

3 Reduced Density Matrices $\quad 8$

4 Polynomial U(6)-invariants of three fermions $\quad 11$ 
6 The canonical region 19

6.1 The canonical region $\Delta \ldots \ldots \ldots \ldots \ldots \ldots \ldots$

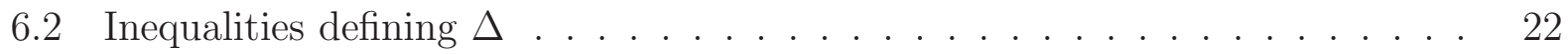

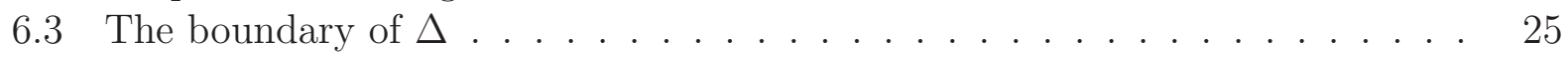

7 Three-fermion canonical form 27

7.1 The canonical form . . . . . . . . . . . . . . . . . 27

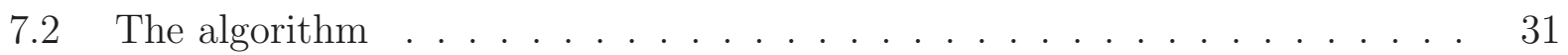

8 Real and quasi-real pure fermionic states 33

9 A new canonical form for pure three-qubit states 34

A The polynomial $f(t) \quad 36$

\section{Introduction}

The underlying resource, which makes quantum information processing more powerful than its classical counterpart, is quantum entanglement. The quantum correlations among a number of players can be much stronger than any classical correlation. Quantum entanglement, as a correlation property, should be preserved in certain ways when only 'local' operations are performed. One of the central tasks of entanglement theory is to classify entanglement types with respect to local operations.

More explicitly, for an $N$-particle state $|\psi\rangle$ in the Hilbert space $\otimes^{N}\left(\mathbb{C}^{M}\right)$, a local operation of the form $\bigotimes_{i=1}^{N} A_{i}$ acting on $|\psi\rangle$ preserves certain entanglement properties of $|\psi\rangle$. The two most studied cases are (i) $A_{i} \in \mathrm{U}(M)$, i.e. all $A_{i}$ are unitary, where all the entanglement properties of $|\psi\rangle$ are preserved; (ii) $A_{i} \in \mathrm{GL}(M, \mathbb{C})$, where entanglement properties of $|\psi\rangle$ under local operation and classical communication (SLOCC) are preserved [1, 2, 3]. As throughout the paper we use the general linear group only over the field of complex numbers, for simplicity we will write $\mathrm{GL}(M, \mathbb{C})$ as $\mathrm{GL}(M)$. Note that $|\psi\rangle$ is non-entangled if and only if it has the form $\left|v_{1}\right\rangle \otimes\left|v_{2}\right\rangle \otimes \cdots \otimes\left|v_{N}\right\rangle$ for some $\left|v_{i}\right\rangle \in \mathbb{C}^{M}$. One of the central tasks in the study of these $N$-party systems is to classify different states, i.e., the orbits under these local Lie groups.

Entanglement of identical particle systems (e.g., fermions and bosons) has also been extensively studied [4, 5, 6, 7, 8, 9], where the concept of entanglement is explored in a mathematical structure different from the tensor product structure of the Hilbert space $\otimes^{N}\left(\mathbb{C}^{M}\right)$. The Hilbert space is now the symmetric power $\vee^{N}\left(\mathbb{C}^{M}\right)$ for bosonic systems, and the exterior power $\wedge^{N}\left(\mathbb{C}^{M}\right)$ for fermionic systems, which can be identified with the subspace of the symmetric and the antisymmetric tensors in $\otimes^{N}\left(\mathbb{C}^{M}\right)$, respectively.

The non-entangled states have the form $|v\rangle^{\vee N}$ in the bosonic case [8, 4, 9], and the form of a Slater determinant $\left|v_{1}\right\rangle \wedge\left|v_{2}\right\rangle \wedge \ldots \wedge\left|v_{N}\right\rangle$ in the fermionic case [7]. Then an LU operation is 
an element $U$ of the unitary group $\mathrm{U}(M)$ acting on the states as the operator $U^{\otimes N}$. Similarly, an SLOCC operation $A$ corresponds to an element of the group GL( $M)$.

In this work we consider the fermionic system with 3 fermions with 6 single particle states, i.e. $N=3$ and $M=6$. We denote by $\wedge^{3}\left(\mathbb{C}^{6}\right) / \mathrm{U}(6)$ the set of $\mathrm{U}(6)$-orbits in $\wedge^{3}(V)$, and define similarly the quotient set $\otimes^{3}\left(\mathbb{C}^{2}\right) /\left((\mathrm{U}(2) \times \mathrm{U}(2) \times \mathrm{U}(2)) \rtimes S_{3}\right)$. Our first main result is to establish the following natural bijective correspondence:

$$
\wedge^{3}\left(\mathbb{C}^{6}\right) / \mathrm{U}(6) \leftrightarrow \otimes^{3}\left(\mathbb{C}^{2}\right) /\left((\mathrm{U}(2) \times \mathrm{U}(2) \times \mathrm{U}(2)) \rtimes S_{3}\right)
$$

To be more precise, choose the orthonormal basis vectors of $V=\mathbb{C}^{6}$ to be $|i\rangle, i=1, \ldots, 6$ and set $e_{i j k}=|i\rangle \wedge|j\rangle \wedge|k\rangle$. From these basis vectors we form three pairs $\{|i\rangle,|i+1\rangle\}$, $i=1,3,5$, and introduce three subspaces

$$
V_{1}=\operatorname{span}\{|1\rangle,|2\rangle\}, \quad V_{2}=\operatorname{span}\{|3\rangle,|4\rangle\}, \quad V_{3}=\operatorname{span}\{|5\rangle,|6\rangle\}
$$

We define the so-called single occupancy vector (SOV) subspace $W$ by

$$
W:=V_{1} \wedge V_{2} \wedge V_{3} \subset \wedge^{3}(V)
$$

It was shown in [10] that any $|\psi\rangle \in \wedge^{3}(V)$ is LU-equivalent to a state $|\phi\rangle \in W$. The subspace $W$ can be identified with the Hilbert space of three qubits by the isometry $W \rightarrow \mathbb{C}^{2} \otimes \mathbb{C}^{2} \otimes \mathbb{C}^{2}$ which is defined by

$$
e_{i+1, j+3, k+5} \mapsto|i j k\rangle, \quad i, j, k \in\{0,1\} .
$$

This identifies $|\psi\rangle \in W$ with a three-qubit state $|\phi\rangle$ in a one-to-one manner. The subgroup $G$ of $\mathrm{U}(6)$ which leaves the subspace $W$ globally invariant is the semidirect product

$$
G:=(\mathrm{U}(2) \times \mathrm{U}(2) \times \mathrm{U}(2)) \rtimes S_{3},
$$

where the symmetric group $S_{3}$ permutes the three copies of $\mathrm{U}(2)$. Given a $\mathrm{U}(6)$-orbit $\mathcal{O} \subseteq$ $\wedge^{3}\left(\mathbb{C}^{6}\right)$, the intersection $\mathcal{O}^{\prime}:=\mathcal{O} \cap W$ is a single $G$-orbit. Conversely, any $G$-orbit in $W$ is contained in a unique $\mathrm{U}(6)$-orbit $\mathcal{O}$. Hence, the correspondence in Eq. (1) is indeed natural. In terms of quantum entanglement theory, this means that there is a one-to-one correspondence between the LU orbits of $N=3, M=6$ fermionic states and the orbits of three-qubit states under LU transformations and qubit permutations. Moreover we show that, when the two quotient sets in Eq. (11) are equipped with quotient topologies, then our one-to-one correspondence is a homeomorphism. There is no hope to generalize these results to the case $N \geq 4, M=2 N$ because there exist pure fermionic states in $\wedge^{4}\left(\mathbb{C}^{M}\right)$ which are not single occupancy states [10].

Our main tool is the powerful invariant theory. In Sec. 4 we construct a minimal set of homogeneous generators of the algebra $\mathcal{A}$ of polynomial $\mathrm{U}(6)$-invariants, which consists of six primary generators, which are algebraically independent, and an additional secondary generator. In Sec. 5 we recall the known results about the algebra of polynomial LUinvariants of three qubits, and construct a minimal set of homogeneous generators for its 
subalgebra $\mathcal{B}$ consisting of the invariants that are additionally fixed by qubit permutations. In Theorem 6 we show that these two algebras are isomorphic as graded algebras, which leads to the result of Eq. (1).

Based on the full knowledge of the algebra $\mathcal{A}$, we move further to construct a canonical form for the fermionic states with $N=3$ and $M=6$. This is the second main result of the paper. That is, any state in $\wedge^{3}\left(\mathbb{C}^{6}\right)$ is LU-equivalent to a state

$$
|\psi\rangle=a e_{235}+b e_{145}+c e_{136}+d e_{246}+z e_{135},
$$

where $a \geq b \geq c \geq 0, z=x+i y$ with $x, y$ real, $x \geq 0$, and $d$ is the maximum of $|\langle\alpha \wedge \beta \wedge \gamma \mid \psi\rangle|$ taken over all unit vectors $|\alpha \wedge \beta \wedge \gamma\rangle$. We denote by $\Delta$ the spherical region consisting of all states $|\psi\rangle$ of that form. We show that $\Delta$ is a closed connected region and that its relative interior $\Delta^{0}$ is dense in $\Delta$. Moreover, we show that if two different states in $\Delta$ are LU-equivalent then they must both lie on the boundary of $\Delta$, see Theorem 21 for more details.

By using the one-to-one correspondence in Eq. (1) and the canonical form for the fermionic states, we construct a new canonical form for three-qubit pure states (without any qubit permutations). This is the third main result of the paper. The isometry Eq. (4) maps the fermionic state Eq. (6) to the three-qubit state

$$
|\phi\rangle=a|100\rangle+b|010\rangle+c|001\rangle+d|111\rangle+z|000\rangle .
$$

When $a, b, c, x \geq 0$ and $d=\max _{\alpha, \beta, \gamma}|\langle\alpha, \beta, \gamma \mid \psi\rangle|$, where $|\alpha, \beta, \gamma\rangle$ is any three-qubit product state, Eq. (7) gives a canonical form for three-qubit pure states. This completes the missing case for three-qubit canonical forms discussed in [11, see Sec. 9 for more details.

We believe that our results, on both the relationships between the invariants of Lie groups $\mathrm{U}(6)$ and $G$, and the canonical forms, will not only attract interest from quantum information science community studying entanglement properties, for both distinguishable particle systems and identical particle systems, but also will be of interest solely mathematically. Like other connections between small Lie groups, we certainly believe the simple format of our results will find applications in yet some other area of science.

We organize our paper as follows. In Sec. 2, we review some related work in quantum information theory that studies local orbits, for both distinguishable and identical particle systems. We compare these previous works to our results, which further motivates our work. In Sec. 3, we discuss reduced density matrices (RDMs) for pure states of the $N=3, M=6$ fermionic system, and compare them with RDMs of three-qubit systems. The spectra of these RDMs, which are obviously invariant under $\mathrm{U}(6)$, will be later used to build some of the invariants. In Sec. 4, we discuss the algebra of polynomial U(6) invariants of the $N=3, M=6$ fermionic system, and in Sec. 5 we consider the algebra of the symmetric polynomial invariants of three qubits. These two algebras are shown to be isomorphic as graded algebras. In Sec. 6 8 we study the canonical form for pure states of the $N=3$, $M=6$ fermionic system. Finally, in Sec. 9 we present a new canonical form for three-qubit pure states. 


\section{Related Work}

\subsection{Systems of distinguishable particles}

It is well-known that in the simplest case of $N=2$ particles, any state $|\psi\rangle$ in $\mathbb{C}^{M} \otimes \mathbb{C}^{M}$ is LU-equivalent to a state in the canonical form given by the Schmidt decomposition

$$
|\psi\rangle=\sum_{i=1}^{M} \sqrt{\lambda_{i}}|i\rangle \otimes|i\rangle,
$$

where the states $|i\rangle, i=1, \ldots, M$ form an orthonormal basis of $\mathbb{C}^{M}$, and $\lambda_{1} \geq \cdots \geq \lambda_{M} \geq 0$. Thus states with different Schmidt coefficients $\lambda_{i}$ will generically be LU-inequivalent. The case $N=3$ turns out to be much more complicated, as no direct generalization of the Schmidt decomposition is available. A canonical form for LU orbits of three qubits was obtained in [11] as

$$
|\psi\rangle=\eta_{0} e^{i \phi}|000\rangle+\eta_{1}|001\rangle+\eta_{2}|100\rangle+\eta_{3}|110\rangle+\eta_{4}|111\rangle,
$$

where the coefficients $\eta_{i}$ are real and nonnegative. However, no canonical form has ever been found for any other $N \geq 3$ system.

The problem of classifying SLOCC orbits was solved, in some other cases of small systems, thanks to the larger group $\mathrm{GL}(M)^{\times N}$ compared to $\mathrm{U}(M)^{\times N}$. It is well-known that threequbit pure states can be entangled in two-inequivalent ways [1], i.e.,

$$
\begin{gathered}
|G H Z\rangle=\frac{1}{\sqrt{2}}(|000\rangle+|111\rangle) \\
\text { and }|W\rangle=\frac{1}{\sqrt{3}}(|001\rangle+|010\rangle+|100\rangle) \text {. }
\end{gathered}
$$

Four-qubit SLOCC equivalence classes have also been identified, however, now infinitely many orbits exist [12, 13]. SLOCC equivalence classes for other cases have also been discussed, for instance the $2 \times M \times N$ states investigated by the range criterion [2], multipartite symmetric states by the tensor rank [3], and multiqubit symmetric states by locally identical operators [14]. In all these investigations invariant theory plays an important role.

Recently, a connection of SLOCC classification with the theory of the quantum marginal problem has been studied [15, 16, 17]. It was shown that the set of vectors with entries given by the eigenvalues of the one particle reduced density matrices is a convex polytope (namely the entanglement polytope), for the set of states in any SLOCC equivalence class. Further, it was shown that there is only a finite number of polytopes for any $N$ and $M$, compared to an in general infinite number of SLOCC orbits. Hence, the result in [15, 16, 17] provides a coarse-grained version of the SLOCC classification. In identifying these polytopes, invariant theory also plays a crucial role. 


\subsection{Fermionic systems}

In the case $N=2$ and even local dimension $M=2 K$, canonical forms for LU orbits similar to the Schmidt decomposition have been obtained for both bosonic and fermionic systems [5, 7, 8]. The bosonic state is LU-equivalent to a state which has exactly the same form as Eq. (8). It means that we have a one-to-one correspondence $\vee^{2}\left(\mathbb{C}^{M}\right) / \mathrm{U}(M) \leftrightarrow$ $\left(\mathbb{C}^{M} \otimes \mathbb{C}^{M}\right) /(\mathrm{U}(M) \times \mathrm{U}(M))$. The fermionic case is a bit different as any fermionic state is LU-equivalent to a special form of Eq. (8)

$$
|\psi\rangle=\sum_{i=1}^{K} \sqrt{\lambda_{i}}\left|\alpha_{i}\right\rangle \wedge\left|\beta_{i}\right\rangle
$$

where $\left\langle\alpha_{i} \mid \alpha_{j}\right\rangle=\left\langle\beta_{i} \mid \beta_{j}\right\rangle=\delta_{i j}$, and $\left\langle\alpha_{i} \mid \beta_{j}\right\rangle=0$. This means that we have a one-to-one correspondence

$$
\wedge^{2}\left(\mathbb{C}^{M}\right) / \mathrm{U}(M) \leftrightarrow\left(\mathbb{C}^{K} \otimes \mathbb{C}^{K}\right) /(\mathrm{U}(K) \times \mathrm{U}(K)) .
$$

This fact is indeed known, see e.g., [10].

For the case $N \geq 3$, there is no generalization of the Schmidt decomposition. So far no nontrivial canonical forms of LU orbits for $N \geq 3$ bosonic/fermionic systems have been identified. By "non-trivial" we mean that there are some trivial cases which can be treated easily. For instance, for bosonic systems with $M=2$, the LU group is just $\mathrm{U}(2)$. For fermionic systems, due to the particle-hole duality, only the cases $M \geq 2 N$ are of interest.

The simplest nontrivial fermionic system with $N=3$ and $M=6$, i.e., three fermions with six single particle states, has attracted much attention recently. Its SLOCC orbits have been completely classified, and it turns out that there is a surprising link to the SLOCC orbits of three-qubits [18. That is, there is a one-to-one correspondence between the SLOCC orbits of these two very different systems

$$
\wedge^{3}\left(\mathbb{C}^{6}\right) / \mathrm{GL}(6) \leftrightarrow\left(\mathbb{C}^{2} \otimes \mathbb{C}^{2} \otimes \mathbb{C}^{2}\right) /\left((\mathrm{GL}(2) \times \mathrm{GL}(2) \times \mathrm{GL}(2)) \rtimes S_{3}\right),
$$

which is yet another accidental coincidence involving small Lie groups. For several $N$ and $M$ fermionic systems with $N \geq 3$, the SLOCC orbits in $\wedge^{N}\left(\mathbb{C}^{M}\right)$ were classified as early as 1931 in the context of multilinear algebra [19]. The SLOCC orbits and polynomial invariants of three qubits were studied in detail in 1999 [20], in the context of $2 \times 2 \times 2$ complex matrices. The fact that there is a natural correspondence between these orbits and the SLOCC orbits of the fermionic system $\wedge^{3}\left(\mathbb{C}^{6}\right)$ is also pointed out in the same reference.

Studies of the $N$-representability problem [21] suggest some further connection of the orbits of the $N=3, M=6$ fermionic system with the orbits of three-qubit system, but in a more complicated situation where the LU orbits are considered. Because of the smaller group $\mathrm{U}(6)$ compared to $\mathrm{GL}(6)$, one needs to deal with many more (in fact infinitely many) orbits. It was shown that, if one arranges the eigenvalues $\lambda_{i}$ of the one-particle RDM of any pure $N=3, M=6$ fermionic state $|\psi\rangle$ in a non-increasing order as $\lambda_{1} \geq \lambda_{2} \geq \cdots \geq \lambda_{5} \geq \lambda_{6}$, then $\lambda_{i}+\lambda_{7-i}=1$ for $i=1,2,3[10,22,23,24,25]$. This indicates that there is always a representative of each LU orbit which adopts a special form, namely the single-occupancy form. 
However, one key question remained unanswered: for a given $N=3, M=6$ fermionic state $|\psi\rangle$, can any two of its LU-equivalent states in $W$ correspond to two LU-inequivalent three-qubit states under the LU group $\mathrm{U}(2) \times \mathrm{U}(2) \times \mathrm{U}(2)$ and the permutation of qubits? In other words, is there a one-to-one correspondence between the $\mathrm{LU}$ orbits of $N=3, M=6$ fermionic states and the three-qubit states, just like in the SLOCC case in (13)? This question is answered affirmatively by (11) and Theorem 6 in Sec. 5. Although (11) and (13) are similar, we emphasize that the former relation is much more important because many physical properties of three-qubit pure states are invariant under the group $\mathrm{U}(2) \times \mathrm{U}(2) \times \mathrm{U}(2)$, but not under $\mathrm{GL}(2) \times \mathrm{GL}(2) \times \mathrm{GL}(2)$. Such properties include entanglement measures (e.g., geometric measure of entanglement in Sec. 2.3), eigenvalues of RDMs and so on. Moreover, the group $\mathrm{U}(2) \times \mathrm{U}(2) \times \mathrm{U}(2)$ is realizable with probability one in experiment, while $\mathrm{GL}(2) \times \mathrm{GL}(2) \times \mathrm{GL}(2)$ corresponding to SLOCC can be realized only with a nonvanishing probability.

Note that when talking about three-qubit orbits, one has to take into account the qubit permutations by the symmetric group $S_{3}$. This is because, in the fermionic case, qubit permutations correspond to permuting the subspaces $V_{1}, V_{2}, V_{3}$, which preserve $W$. On the other hand, in considering the three-qubit LU orbits such as Eq. (9), the qubit permutations were not taken into account [11. In fact, two states given by Eq. (91) with different parameters $a, b, c$ may correspond to the same orbit under $(\mathrm{U}(2) \times \mathrm{U}(2) \times \mathrm{U}(2)) \rtimes S_{3}$. So Eq. (9) is no longer a canonical form in this case. As permutation of qubits does not change the main entanglement properties of quantum states, it should be taken into account for any task involving entanglement classification.

\subsection{Geometric measure of entanglement}

In quantum information science the geometric measure of entanglement (GME) is a known multipartite entanglement measure [26]. For $N$-partite pure states $|\psi\rangle \in \otimes_{i=1}^{N} \mathcal{H}_{i}$, the GME is defined as [27]

$$
G(\psi)=1-\Lambda^{2}(\psi)
$$

where $\Lambda(\psi)=\max _{\left|a_{1}, \ldots, a_{N}\right\rangle}\left|\left\langle a_{1}, \ldots, a_{N} \mid \psi\right\rangle\right|$, and $\left|a_{i}\right\rangle \in \mathcal{H}_{i}$ are unit vectors. For fermionic system, we can similarly define the GME for $N$-vectors as

$$
G_{f}(\psi)=1-\Lambda_{f}^{2}(\psi)
$$

where $\Lambda_{f}(\psi)=\max _{\left|b_{1} \wedge \ldots \wedge b_{N}\right\rangle}\left|\left\langle b_{1} \wedge \ldots \wedge b_{N} \mid \psi\right\rangle\right|$, and $\left|b_{1} \wedge \cdots \wedge b_{N}\right\rangle$ are unit vectors. In the case $N=3, M=6$, for any pure fermionic state $|\psi\rangle$ we will define the quantity $\mu(\psi)$ to be the maximum overlap $|\langle\alpha \wedge \beta \wedge \gamma \mid \psi\rangle|$ over all decomposable three-vectors $|\alpha \wedge \beta \wedge \gamma\rangle$ of unit norm in Definition 9. In particular, $\Lambda_{f}(\psi)=\mu(\psi)$ for any 3-vector $|\psi\rangle \in W$, where $W$ is the SOV space defined in (3). By using the embedding relation (16) and Lemma 10, we can obtain $\Lambda(\psi)=6^{-1 / 2} \Lambda_{f}(\psi)$ for $|\psi\rangle \in W$.

Since $\mu$ does not change under local unitary operations, it follows that if $\mu(\phi) \neq \mu(\psi)$ then $|\phi\rangle$ and $|\psi\rangle$ are not LU-equivalent. Recall from Eq. (1) that when $N=3, M=6$ 
there is a one-to-one correspondence between the LU orbits of fermionic states and the LU plus permutations orbits of three-qubit states. By (14), (15), and Lemma 10, computing the GME of $|\psi\rangle \in \wedge^{3}\left(C^{6}\right)$ is equivalent to the same task for three-qubit pure states.

In recent years, the GME has been used in the context of many different aspects in quantum information. First, most multipartite states have been shown to be too entangled to implement measurement-based quantum computing via GME [28]. GME is also important for studying multiple-prover quantum Merlin-Arthur games [29] and weak multiplicativity for random quantum channels [30]. Second, there have been efforts to search for the maximally entangled multiqubit states with respect to the GME [31, 32]. Research has also been carried out over the additivity, and computation of GME for Dicke, Smolin, stabilizer, and antisymmetric states [33, 34. Third, computing the GME is helpful for the understanding of other important entanglement measures including the entanglement of formation [27], relative entropy of entanglement, and entanglement of robustness [35, 34]. Fourth, the GME is also related to the long-standing open problems on symmetrical-informationally-complete (SIC)-POVM and mutually unbiased bases (MUB) [36].

Thus studying the GME is important, however it has been proved to be hard even for three-qubit pure states [31. Our Lemma 12 gives a general method for computing the quantity $\mu$ (and the GME) of the linear superposition of two states with known values of $\mu$. Next, Theorem 16 gives a collection of inequalities which cut out a spherical region $\Delta$, by which one can decide whether a given positive number is the value of $\mu$ of some three-qubit pure state. Third, we show that the maximally entangled three-qubit pure state with respect to the GME is the $W$ state, see Proposition [19, This gives an independent proof of the main result of [31]. Fourth, Proposition 20 shows that the region $\Delta$ provides the canonical form for three-fermionic states $|\psi\rangle$, in which $d=\mu(\psi)$ is one of the coordinates. Fifth, we give an algorithm to compute the GME for three-qubit states in Sec. 7.2.

\section{$3 \quad$ Reduced Density Matrices}

We consider $N$-vectors of an $M$-dimensional Hilbert space $V$ as antisymmetric tensors. We shall use the isometric embedding

$$
\left|v_{1} \wedge v_{2} \wedge \cdots \wedge v_{N}\right\rangle \rightarrow \frac{1}{\sqrt{N !}} \sum_{\sigma \in S_{N}} \operatorname{sgn}(\sigma)\left|v_{\sigma(1)}, v_{\sigma(2)}, \ldots, v_{\sigma(N)}\right\rangle
$$

Thus we may consider $\wedge^{N}(V)$ as a subspace of $\otimes^{N}(V)$. Consequently, we may apply the partial trace operators to the density matrix $\rho=|\psi\rangle\langle\psi|$. Although $\rho$ acts on $\otimes^{N}(V)$, its support (i.e., range) is contained in $\wedge^{N}(V)$ and we shall identify $\rho$ with its restriction to $\wedge^{N}(V)$.

The general linear group $\mathrm{GL}:=\mathrm{GL}(V)$ acts on $\mathcal{H}$ by the so called diagonal action:

$$
A \cdot\left(\left|v_{1}\right\rangle \otimes\left|v_{2}\right\rangle \otimes \cdots \otimes\left|v_{N}\right\rangle\right)=A\left|v_{1}\right\rangle \otimes A\left|v_{2}\right\rangle \otimes \cdots \otimes A\left|v_{N}\right\rangle, \quad A \in \mathrm{GL}, \quad\left|v_{i}\right\rangle \in V .
$$

In other words, $A \in$ GL acts on $\mathcal{H}$ as $\otimes^{N} A$. Similarly, we have the action of GL on $\wedge^{N} V$ where $A \in \mathrm{GL}$ acts as $\wedge^{N} A$, i.e., we have 


$$
A \cdot\left|v_{1} \wedge \cdots \wedge v_{N}\right\rangle=\left|A v_{1} \wedge \cdots \wedge A v_{N}\right\rangle
$$

These actions can be restricted to the unitary group $\mathrm{U}(V)$ of $V$. We shall say that two $N$-vectors $|\phi\rangle$ and $|\psi\rangle$ are equivalent if they belong to the same GL-orbit, i.e., $|\psi\rangle=A \cdot|\phi\rangle$ for some $A \in \mathrm{GL}$. We shall also say that they are unitarily equivalent or $L U$-equivalent if $A$ can be chosen to be unitary.

In this paper we consider only the case where $M=6$ and $N=3$, that is, three fermions with six single particle states. We have the following result from [10].

Lemma 1 Any three-fermion pure state with six single particle states is $L U$-equivalent to

$$
|\psi\rangle=a e_{235}+b e_{145}+c e_{136}+d e_{246}+z e_{135},
$$

where $e_{i j k}=|i\rangle \wedge|j\rangle \wedge|k\rangle$, the coefficients $a, b, c, d \geq 0, z \in \mathbb{C}$, and $\|\psi\|^{2}=a^{2}+b^{2}+c^{2}+$ $d^{2}+|z|^{2}=1$.

The symmetric group $S_{3}$ is generated by the 3-cycle $\sigma=(123)$ and the transposition $\tau=(12)$. We embed $S_{3}$ into the unitary group $\mathrm{U}(6)$ as follows:

$$
\sigma=\left(\begin{array}{ccc}
0 & 0 & I_{2} \\
I_{2} & 0 & 0 \\
0 & I_{2} & 0
\end{array}\right), \quad \tau=-\left(\begin{array}{ccc}
0 & I_{2} & 0 \\
I_{2} & 0 & 0 \\
0 & 0 & I_{2}
\end{array}\right)
$$

where $I_{2}$ is the $2 \times 2$ identity matrix.

Note that $S_{3}$ permutes the subspaces $V_{1}, V_{2}, V_{3}$. Consequently, under the action of $\mathrm{U}(6)$ on $\wedge^{3}(V), S_{3}$ leaves invariant the subspace $W$. Moreover, $S_{3}$ permutes the basis vectors $e_{135}, e_{145}, e_{136}, e_{146}, e_{235}, e_{245}, e_{236}, e_{246}$ of $W$. The 3 -cycle $\sigma$ fixes $e_{135}$ and $e_{246}$, and sends $e_{145} \rightarrow e_{136} \rightarrow e_{235} \rightarrow e_{145}$ and $e_{146} \rightarrow e_{236} \rightarrow e_{245} \rightarrow e_{146}$. The transposition $\tau$ fixes $e_{135}$, $e_{136}, e_{245}, e_{246}$, and sends $e_{145} \rightarrow e_{235} \rightarrow e_{145}$ and $e_{146} \rightarrow e_{236} \rightarrow e_{146}$. It follows that under this action, $S_{3}$ preserves the real 6-dimensional subspace $W_{6}$ with basis

$$
\left\{e_{235}, e_{145}, e_{136}, e_{246}, e_{135}, i e_{135}\right\}
$$

In terms of the coordinates $a, b, c, d, z$, the action of $\sigma$ and $\tau$ on $W_{6}$ is given by

$$
\sigma \cdot(a, b, c, d, z)=(c, a, b, d, z) \quad \text { and } \quad \tau \cdot(a, b, c, d, z)=(b, a, c, d, z) .
$$

(This is the reason why we introduced the factor -1 in the definition of the matrix of $\tau$.) Thus we can assume that $a \geq b \geq c \geq 0$ and $d \geq 0$.

To compute the 1-body RDM for $|\psi\rangle$, we use a different normalization i.e. it has trace 3 , as used by chemists [22]. Thus we set $\rho^{(1)}=3 \rho_{1}$, where $\rho_{1}$ is the normalized 1-RDM. 
A computation shows that $\rho^{(1)}=R_{a} \oplus R_{b} \oplus R_{c}$, where

$$
\begin{aligned}
R_{a} & =\left(\begin{array}{cc}
b^{2}+c^{2}+|z|^{2} & a z \\
a z^{*} & a^{2}+d^{2}
\end{array}\right), \\
R_{b} & =\left(\begin{array}{cc}
c^{2}+a^{2}+|z|^{2} & b z \\
b z^{*} & b^{2}+d^{2}
\end{array}\right), \\
R_{c} & =\left(\begin{array}{cc}
a^{2}+b^{2}+|z|^{2} & c z \\
c z^{*} & c^{2}+d^{2}
\end{array}\right) .
\end{aligned}
$$

For $x=a, b, c$ let $D_{x}=\operatorname{det} R_{x}$, i.e.,

$$
\begin{aligned}
& D_{a}=\left(b^{2}+c^{2}\right)\left(a^{2}+d^{2}\right)+d^{2}|z|^{2}, \\
& D_{b}=\left(c^{2}+a^{2}\right)\left(b^{2}+d^{2}\right)+d^{2}|z|^{2}, \\
& D_{c}=\left(a^{2}+b^{2}\right)\left(c^{2}+d^{2}\right)+d^{2}|z|^{2} .
\end{aligned}
$$

Since $\operatorname{Tr} R_{x}=1$ and $R_{x} \geq 0$, we have $D_{x} \in[0,1 / 4]$, and the eigenvalues of $R_{x}$ can be written as $\lambda_{x}$ and $1-\lambda_{x}$ with $\lambda_{x}=\left(1+\sqrt{1-4 D_{x}}\right) / 2 \in[1 / 2,1]$. Let us denote the eigenvalues of $\rho^{(1)}$ arranged in decreasing order as $\lambda_{1} \geq \lambda_{2} \geq \cdots \geq \lambda_{5} \geq \lambda_{6}$. Then $\lambda_{i}$ and $\lambda_{7-i}$ are the eigenvalues of the same block $R_{x}$ of $\rho^{(1)}$. Thus we obtain that if the $\lambda_{i}$ are arranged in decreasing order, then $\lambda_{i}+\lambda_{7-i}=1$ for $i=1,2,3$ (this fact is proved in [21, 22, 24, 25] using other methods).

Next we consider the 2-particle RDM $\rho_{1,2}$. This is a $\left(\begin{array}{l}6 \\ 2\end{array}\right) \times\left(\begin{array}{l}6 \\ 2\end{array}\right)$ matrix, which is also block diagonal with three $4 \times 4$ blocks,

Block 1: coordinates $(1,3),(1,4),(2,3),(2,4)$, the corresponding block in $\rho_{1,2}$ is denoted by $\rho_{1,2}^{[1]}$,

Block 2: coordinates $(1,5),(1,6),(2,5),(2,6)$, the corresponding block in $\rho_{1,2}$ is denoted by $\rho_{1,2}^{[2]}$,

Block 3: coordinates $(3,5),(3,6),(4,5),(4,6)$, the corresponding block in $\rho_{1,2}$ is denoted by $\rho_{1,2}^{[3]}$,

and a zero $3 \times 3$ block at the coordinates $(1,2),(3,4),(5,6)$.

Using Eq. (18), the three non-zero blocks are given by

$$
\begin{aligned}
\rho_{1,2}^{[1]} & =\left(\begin{array}{cccc}
c^{2}+|z|^{2} & b z & a z & c d \\
b z^{*} & b^{2} & a b & 0 \\
a z^{*} & a b & a^{2} & 0 \\
c d & 0 & 0 & d^{2}
\end{array}\right), \\
\rho_{1,2}^{[2]} & =\left(\begin{array}{cccc}
b^{2}+|z|^{2} & c z & a z & b d \\
c z^{*} & c^{2} & a c & 0 \\
a z^{*} & a c & a^{2} & 0 \\
b d & 0 & 0 & d^{2}
\end{array}\right),
\end{aligned}
$$




$$
\rho_{1,2}^{[3]}=\left(\begin{array}{cccc}
a^{2}+|z|^{2} & c z & b z & a d \\
c z^{*} & c^{2} & b c & 0 \\
b z^{*} & b c & b^{2} & 0 \\
a d & 0 & 0 & d^{2}
\end{array}\right)
$$

Note that for $|\psi\rangle$ given in Eq. (18), $|\psi\rangle \in W$ is a SOV state. So $|\psi\rangle$ can be identified as a qubit state for qubits $A, B, C$, via the map $e_{i+1, j+3, k+5} \rightarrow|i j k\rangle, i, j, k \in\{0,1\}$, i.e.

$$
|\psi\rangle=a|100\rangle+b|010\rangle+c|001\rangle+d|111\rangle+z|000\rangle
$$

Then we have

$$
\begin{aligned}
\rho_{1,2}^{[1]} & =\rho_{A B}=\operatorname{Tr}_{C}|\psi\rangle\langle\psi|, \\
\rho_{1,2}^{[2]} & =\rho_{A C}=\operatorname{Tr}_{B}|\psi\rangle\langle\psi|, \\
\rho_{1,2}^{[3]} & =\rho_{B C}=\operatorname{Tr}_{A}|\psi\rangle\langle\psi| .
\end{aligned}
$$

\section{Polynomial U(6)-invariants of three fermions}

We continue to use the real 6-dimensional subspace $W_{6} \subseteq \wedge^{3}(V)$ introduced in Section 3 , We shall write an arbitrary $|\psi\rangle \in W_{6}$ as in Eq. (18), and use $a, b, c, d$ and $z=x+i y$ as its coordinates (with $x$ and $y$ real).

We denote by $\mathrm{U}(6)$ the unitary group of $V$. We set $\rho=|\psi\rangle\langle\psi|$ where

$$
|\psi\rangle=\sum_{1 \leq i<j<k \leq 6} \xi_{i j k} e_{i j k}, \quad \xi_{i j k} \in \mathbb{C}
$$

is an arbitrary 3 -vector. Since we work with non-normalized pure states we should specify how we normalize the density matrix $\rho$ and its one- and two-body RDMs $\rho_{1}:=\operatorname{Tr}_{2,3}(\rho)$ and $\rho_{1,2}:=\operatorname{Tr}_{3}(\rho)$. We shall require that

$$
\operatorname{Tr} \rho=\operatorname{Tr} \rho_{1}=\operatorname{Tr} \rho_{1,2}=\|\psi\|^{2}=\sum_{i<j<k}\left|\xi_{i j k}\right|^{2}
$$

Thus, if $|\psi\rangle$ is a unit vector then the density matrices $\rho, \rho_{1}$, and $\rho_{1,2}$ all have trace 1 . Note that this normalization is different from the one used in Section 3.

Let us introduce the $\mathrm{U}(6)$ or LU-invariants of $\wedge^{3}(V)$. To begin, we have to consider $\wedge^{3}(V)$ as a real vector space, and our invariants will belong to the algebra $\mathcal{P}_{\mathbb{R}}$ of complex-valued polynomials defined on this real vector space. We shall denote this algebra of invariants by $\mathcal{P}_{\mathbb{R}}^{U}$. After complexification, we obtain the representation of $\operatorname{GL}(6)$ on $\mathbb{C} \otimes_{\mathbb{R}} \wedge^{3}(V)$. This tensor product decomposes into a direct sum of two complex holomorphic representations of $\mathrm{GL}(6)$, namely $\wedge^{3}(V)$ and its complex conjugate representation. However, since $\mathrm{U}(6)$ is a compact group, the complex conjugate representation is isomorphic to the dual representation. These two irreducible representations of GL(6) are not isomorphic, but they become 
isomorphic when restricted to $\mathrm{SL}(6)$ (again, by $\mathrm{SL}(6)$ we mean $\mathrm{SL}(6, \mathbb{C})$ ). This means that $\mathbb{C} \otimes_{\mathbb{R}} \wedge^{3}(V)$ is the direct sum of two copies of the fundamental representation of SL(6) on $\wedge^{3}(V)$. Let us denote by $\mathcal{P}$ the algebra of complex holomorphic polynomials on $\mathbb{C} \otimes_{\mathbb{R}} \wedge^{3}(V)$. The complexification of $\mathcal{P}_{\mathbb{R}}^{\mathrm{SU}}$ coincides with the algebra $\mathcal{P}^{\mathrm{SL}}$ of holomorphic polynomial $\mathrm{SL}(6)$-invariants on $\mathbb{C} \otimes_{\mathbb{R}} \wedge^{3}(V)$. It is known [37] that this algebra is regular, i.e., it is a polynomial algebra (in 7 variables).

Due to the direct decomposition of $\mathbb{C} \otimes_{\mathbb{R}} \wedge^{3}(V)$, the algebra $\mathcal{P}$ is bigraded, and the same is true for its subalgebra $\mathcal{P}^{\mathrm{SL}}$. From [37, Table 2a] we know that the 7 bihomogeneous generators of $\mathcal{P}^{\mathrm{SL}}$ have bidegrees $(1,1),(4,0),(3,1),(2,2),(1,3),(0,4),(3,3)$. Hence, the bigraded Poincaré series of $\mathcal{P}^{\mathrm{SL}}$ is

$$
f(s, t)=\frac{1}{(1-s t)\left(1-s^{4}\right)\left(1-s^{3} t\right)\left(1-s^{2} t^{2}\right)\left(1-s t^{3}\right)\left(1-t^{4}\right)\left(1-s^{3} t^{3}\right)} .
$$

It is not hard to compute the bigraded Poincare series of $\mathcal{P}^{\mathrm{GL}}$. We can distinguish the two irreducible submodules of $\mathbb{C} \otimes_{\mathbb{R}} \wedge^{3}(V)$ by taking into account the action of the central torus of GL(6). Thus, we replace $s, t$ with $s z, t z^{-1}$ respectively, and expand $f\left(s z, t z^{-1}\right)$ into the Laurent series with respect to the variable $z$. Then the bigraded Poincaré series of $\mathcal{P}$ GL, say $g(s, t)$, is the constant term of that Laurent series. It is given by the contour integral over the unit circle:

$$
g(s, t)=\frac{1}{2 \pi i} \int f\left(s z, t z^{-1}\right) \frac{\mathrm{d} z}{z} .
$$

By computing this contour integral by standard methods (e.g., the Residue Theorem) and then setting $s=t$, we obtain the ordinary Poincaré series of $\mathcal{P}^{\mathrm{GL}}$ :

$$
\begin{aligned}
g(t, t) & =\frac{1+t^{12}}{\left(1-t^{2}\right)\left(1-t^{4}\right)\left(1-t^{6}\right)\left(1-t^{8}\right)^{2}\left(1-t^{12}\right)} \\
& =1+t^{2}+2 t^{4}+3 t^{6}+6 t^{8}+7 t^{10}+13 t^{12}+16 t^{14}+25 t^{16}+31 t^{18}+46 t^{20}+\cdots
\end{aligned}
$$

This is also the Poincaré series of $\mathcal{P}_{\mathbb{R}}^{U}$.

Any polynomial LU-invariant $f$ is uniquely determined by its restriction $f^{\prime}:=\left.f\right|_{W_{6}}$. These restrictions are polynomials in only 6 real variables, and so calculations with them are much faster than with the full expressions for the invariants which depend on 40 real variables. When convenient, we may specify $f$ by giving explicit expression for its restriction $f^{\prime}$.

Let us now describe the generators of $\mathcal{P}_{\mathbb{R}}^{U}$ that we shall use. The norm square, $M_{1}:=$ $\|\psi\|^{2}$, is the unique invariant of degree 2. By using our normalizations (28), we also have $M_{1}=\operatorname{Tr}(\rho)=\operatorname{Tr}\left(\rho_{1}\right)=\operatorname{Tr}\left(\rho_{1,2}\right)$.

The representation space $\wedge^{3}\left(\mathbb{C}^{6}\right)$ of $\mathrm{GL}(6)$ is a well known regular prehomogeneous vector space, [38, Example 2.5]. We shall denote its relative invariant by $F$. In particular, $F$ is also an $\mathrm{SU}(6)$-invariant, in fact the unique invariant of bidegree $(4,0)$, and its complex conjugate 
is the unique invariant of bidegree $(0,4)$. The invariant $F$ was recently rediscovered by Levay and Vrana who also found a nice explicit formula for it [18, Eq. (14)]. When restricted to $W_{6}$, it has a very simple expression

$$
F^{\prime}=d\left(4 a b c+d z^{2}\right)
$$

The elementary symmetric functions of the determinants $D_{x}$ defined in Eqs. (22)-(24), namely

$$
\begin{aligned}
& M_{2}^{\prime}=D_{a}+D_{b}+D_{c}, \\
& M_{4}^{\prime}=D_{a} D_{b}+D_{b} D_{c}+D_{c} D_{a}, \\
& M_{6}^{\prime}=D_{a} D_{b} D_{c},
\end{aligned}
$$

turn out to be restrictions to $W_{6}$ of polynomial $\mathrm{U}(6)$-invariants which we will denote by $M_{2}$, $M_{4}$, and $M_{6}$, respectively. Explicitly, these three invariants can be defined as follows:

$$
\begin{aligned}
& M_{2}=\frac{3}{2}\left(M_{1}^{2}-3 \operatorname{Tr}\left(\rho_{1}^{2}\right)\right) \\
& M_{4}=\frac{1}{4}\left(3 M_{1}^{4}+2 M_{2}^{2}-4 M_{1}^{2} M_{2}-81 \operatorname{Tr}\left(\rho_{1}^{4}\right)\right) \\
& M_{6}=\frac{1}{6}\left(3 M_{1}^{6}-6 M_{1}^{4} M_{2}+9 M_{1}^{2} M_{2}^{2}-18 M_{1}^{2} M_{4}-2 M_{2}^{3}+6 M_{2} M_{4}-729 \operatorname{Tr}\left(\rho_{1}^{6}\right)\right) .
\end{aligned}
$$

Next we introduce the invariants $M_{3}$ and $M_{5}$ by giving two different expressions for each:

$$
\begin{aligned}
M_{3} & =\frac{3}{2} M_{1} M_{2}-\frac{1}{8}\left\|\nabla M_{2}\right\|^{2} \\
& =3 M_{1}\left(M_{1}^{2}-M_{2}\right)-27 \operatorname{Tr}\left(\rho_{1} \operatorname{Tr}_{2}\left(\rho_{1,2}^{2}\right)\right) \\
M_{5} & =\frac{1}{18}\left(10 M_{2}^{2}+8 M_{1} M_{3}-24 M_{4}-\nabla M_{2} \cdot \nabla M_{3}\right), \\
& =|F|^{2}
\end{aligned}
$$

where $\nabla M_{i}$ denotes the gradient of $M_{i}$ considered as a function of 40 real variables, namely the real and imaginary parts of the $\xi_{i j k}$ in Eq. (27).

We shall see below that the invariants $M_{1}, \ldots, M_{6}$ defined above are the primary invariants of $\mathcal{P}_{\mathbb{R}}^{\mathrm{U}}$. There is only one secondary invariant $M_{7}$ which will be temporarily specified by its restriction $M_{7}^{\prime}$.

For convenience, we introduce the following abbreviations

$$
s_{1}:=a^{2}+b^{2}+c^{2}, \quad s_{2}:=a^{2} b^{2}+a^{2} c^{2}+b^{2} c^{2}, \quad s_{3}:=a^{2} b^{2} c^{2} .
$$


Let us now write explicitly the restriction $M_{i}^{\prime}$ of the above seven invariants:

$$
\begin{aligned}
M_{1}^{\prime}= & s_{1}+d^{2}+|z|^{2}, \\
M_{2}^{\prime}= & 2\left(s_{2}+s_{1} d^{2}\right)+3 d^{2}|z|^{2}, \\
M_{3}^{\prime}= & M_{1}^{\prime}\left(s_{2}+s_{1} d^{2}\right)-6\left(s_{3}+a b c d\left(x^{2}-y^{2}\right)+s_{2} d^{2}\right), \\
M_{4}^{\prime}= & s_{1} s_{3}+s_{2}^{2}+3\left(s_{1} s_{2}-s_{3}\right) d^{2}+4\left(s_{2}+s_{1} d^{2}\right) d^{2}|z|^{2}+\left(s_{1}^{2}+s_{2}+3|z|^{4}\right) d^{4}, \\
M_{5}^{\prime}= & d^{2}\left|4 a b c+d z^{2}\right|^{2}=d^{2}\left(16 s_{3}+8 a b c d\left(x^{2}-y^{2}\right)+d^{2}|z|^{4}\right), \\
M_{6}^{\prime}= & \left(s_{1} s_{2}-s_{3}\right)\left(d^{6}+s_{1} d^{4}+s_{2} d^{2}+s_{3}+3 d^{4}|z|^{2}\right)+\left(s_{1} s_{3}+s_{2}^{2}\right) d^{2}|z|^{2} \\
& +2 s_{2} d^{4}|z|^{4}+\left(s_{1}^{2}+s_{2}+2 s_{1}|z|^{2}+|z|^{4}\right) d^{6}|z|^{2}, \\
M_{7}^{\prime}= & a b c d x y \Phi(a, b, c, d, z),
\end{aligned}
$$

where

$$
\Phi(a, b, c, d, z)=d^{2}\left(d^{2}-s_{1}\right)\left(d^{2}-s_{1}-|z|^{2}\right)-2 a b c d\left(x^{2}-y^{2}\right)-4 s_{3} .
$$

There is an $\mathrm{SU}(6)$-invariant, $J$, of bidegree $(1,3)$ whose restriction to $W_{6}$ is given by

$$
J^{\prime}=2 a b c z+d z^{*}\left(M_{1}^{\prime}-2 d^{2}\right) .
$$

However, in order to uniquely identify $J$, we have to replace $W_{6}$ with the 7 -dimensional real subspace $W_{7} \supset W_{6}$, for which we allow the coordinate $d$ in Eq. (18) to become complex. Thus, we replace $d$ with the complex coordinate $w$. By denoting the restriction of $J$ to $W_{7}$ by $J^{\prime \prime}$, and similarly for $M_{1}$, we have

$$
J^{\prime \prime}=2 a b c z+w^{*} z^{*}\left(M_{1}^{\prime \prime}-2|w|^{2}\right) .
$$

One can verify that

$$
M_{7}=-\frac{1}{8} \Im\left(F J^{2}\right)
$$

We shall indicate later in this section how one can construct the invariant $M_{7}$ without using $J$. As $F$ is an $\mathrm{SU}(6)$-invariant of bidegree $(4,0)$, it follows that $F J^{2}$ is a unitary invariant. Consequently, $M_{7}$ is also a unitary invariant. Moreover, direct computation shows that

$$
|J|^{2}=\frac{1}{3} M_{1}\left(M_{1} M_{2}-2 M_{3}\right)+M_{2}^{2}-4 M_{4}-M_{5}
$$

Theorem 2 The algebra $\mathcal{A}:=\mathcal{P}_{\mathbb{R}}^{\mathrm{U}}$ is generated over $\mathbb{C}$ by the invariants $M_{1}, \ldots, M_{7}$. The first six of these generators are algebraically independent. If $\mathcal{A}_{p}$ is the subalgebra of $\mathcal{A}$ generated by these six polynomials, then $\mathcal{A}$ is a free $\mathcal{A}_{p}$-module with basis $\left\{1, M_{7}\right\}$.

Proof. We have seen that the $M_{i}$ are unitary invariants. The algebraic independence of the polynomials $M_{1}, \ldots, M_{6}$ can be verified by exhibiting a point in $\wedge^{3}(V)$ at which the Jacobian of these polynomials has rank 6 . In fact it suffices to verify this for the restrictions 
$M_{1}^{\prime}, \ldots, M_{6}^{\prime}$. In that case one can use the point in $W_{6}$ with coordinates $a=1, b=2, c=3$, $d=4$, and $x=y=1$. Hence, $\mathcal{A}_{p}$ is isomorphic to the polynomial algebra over $\mathbb{C}$ in six variables. The homogeneous component, say $\mathcal{A}_{p}^{(12)}$, of degree 12 of $\mathcal{A}_{p}$ has dimension 12 , while the one of $\mathcal{A}$ has dimension 13 , see Eq. (31). One can easily verify that $M_{7} \notin \mathcal{A}_{p}^{(12)}$. If $f \in \mathcal{A}_{p} \cap \mathcal{A}_{p} M_{7}$, then $f=g M_{7}$ for some $g \in \mathcal{A}_{p}$. As $\mathcal{A}_{p}$ is a unique factorization domain, it is integrally closed in its field of fractions, say $K$. If $f \neq 0$ then also $g \neq 0$, and we obtain that $M_{7}=f / g \in K$. For convenience, let $h=F J^{2}$ and we point out that $|h|^{2}=h h^{*}=|F|^{2}|J|^{4}=M_{5}|J|^{4}$. Eq. (55) shows that $|J|^{2} \in \mathcal{A}_{p}$, and so $|h|^{2} \in \mathcal{A}_{p}$. By a computer calculation, one can easily check that $h+h^{*}$ belongs to $\mathcal{A}_{p}$. Explicitly, we have

$$
9\left(h+h^{*}\right)=\left(M_{1} M_{2}-2 M_{3}\right)^{2}+18 M_{2}\left(M_{2}^{2}-4 M_{4}-M_{5}\right)+9 M_{1}^{2} M_{5}+144 M_{6} .
$$

This, and the identity $\left(h-h^{*}\right)^{2}=\left(h+h^{*}\right)^{2}-4|h|^{2}$, imply that $M_{7}^{2} \in \mathcal{A}_{p}$, and so $M_{7}$ is integral over $\mathcal{A}_{p}$. As $\mathcal{A}_{p}$ is integrally closed, we must have $M_{7} \in \mathcal{A}_{p}$, which gives a contradiction.

We conclude that $f=0$, and so $\mathcal{A}_{p} \cap \mathcal{A}_{p} M_{7}=0$. Since the Poincaré series of $\mathcal{A}$ is the product of $1+t^{12}$ and the Poincaré series of $\mathcal{A}_{p}$, we must have $\mathcal{A}=\mathcal{A}_{p} \oplus \mathcal{A}_{p} M_{7}$. In particular, the invariants $M_{1}, \ldots, M_{7}$ generate $\mathcal{A}$.

Since the unitary invariants separate the LU-orbits [39, Theorem 3, p. 133], we have the following simple test for LU-equivalence of pure fermionic states.

Corollary 3 Two pure states $|\varphi\rangle,|\psi\rangle \in \wedge^{3}(V)$ are LU-equivalent if and only if $M_{i}(\varphi)=$ $M_{i}(\psi)$ for $i=1, \ldots, 7$.

From Eqs. (48), (55), and (56) we obtain that

$$
\begin{aligned}
12^{4} M_{7}^{2} & =36 M_{5}\left(M_{1}\left(M_{1} M_{2}-2 M_{3}\right)+3 M_{2}^{2}-12 M_{4}-3 M_{5}\right)^{2} \\
& -\left(\left(M_{1} M_{2}-2 M_{3}\right)^{2}+18 M_{2}\left(M_{2}^{2}-4 M_{4}-M_{5}\right)+9 M_{1}^{2} M_{5}+144 M_{6}\right)^{2} .
\end{aligned}
$$

This is the unique algebraic relation (syzygy) among the seven generators $M_{i}$, all other such relations are consequences of this one. Thus we can construct the invariant $M_{7}$ (up to \pm sign) from this formula, and so avoid the use of the invariant $J$. The sign of $M_{7}$ should be chosen to agree with Eq. (50).

We remark that the invariants $M_{1}, \ldots, M_{7}$ take real values only. This follows from the fact that the restrictions $M_{i}^{\prime}$ of the $M_{i}$ are real-valued, see Eqs. (44)-(50). While $M_{7}$ obviously may take both positive and negative values, we claim that $M_{i} \geq 0$ for $i \neq 7$. The claim is evidently valid for $i=1$ and $i=5$, and for $i=2,4,6$ it follows from Eqs. (33)-(35). It remains to prove the claim for $i=3$. It suffices to verify that the formula Eq. (46) can be written as

$$
\begin{aligned}
M_{3}^{\prime}= & x^{2}\left((a b-c d)^{2}+(a c-b d)^{2}+(a d-b c)^{2}\right)+ \\
& y^{2}\left((a b+c d)^{2}+(a c+b d)^{2}+(a d+b c)^{2}\right)+S,
\end{aligned}
$$

where $2 S=\sum\left(\alpha^{2}-\beta^{2}\right)^{2} \gamma^{2}$. The summation is over the 12 ordered pairs $(\{\alpha, \beta\}, \gamma)$ where $\alpha, \beta, \gamma$ are three distinct elements of the set $\{a, b, c, d\}$. We omit the details of this verification. 
Let us also remark that $M_{1} M_{2}-2 M_{3} \geq 0$. Indeed, we have

$$
\frac{1}{3}\left(M_{1}^{\prime} M_{2}^{\prime}-2 M_{3}^{\prime}\right)=\left(2 a b c-d y^{2}\right)^{2}+d^{2}|z|^{2}\left(M_{1}^{\prime}-y^{2}\right)+4 s_{2} d^{2}+d x^{2}\left(4 a b c+d y^{2}\right),
$$

which follows immediately from Eqs. (44)-(46).

We recall that there are exactly four nonzero GL(6)-orbits in $\wedge^{3}(V)$. By analogy with the pure three-qubit states (see [18]) we label these orbits as follows and give their representatives:

(i) (fully) separable: $e_{246}$;

(ii) biseparable: $e_{235}+e_{246}$;

(iii) W type: $e_{235}+e_{145}+e_{136}$;

(iv) GHZ type: $e_{135}+e_{246}$.

We warn the reader that, according to this definition, a separable state is not biseparable. We can determine the type of any state by using the invariants.

Proposition 4 A (non-normalized) state $|\psi\rangle \in \wedge^{3}(V)$ is

(i) separable if and only if $M_{2}(\psi)=0$;

(ii) biseparable if and only if $M_{5}$ and $M_{1} M_{2}-2 M_{3}$ vanish at $|\psi\rangle$, and $M_{2}(\psi)>0$;

(iii) of $W$ type if and only if $M_{5}(\psi)=0$ and $M_{1}(\psi) M_{2}(\psi)-2 M_{3}(\psi)>0$;

(iv) of GHZ type if and only if $M_{5}(\psi)>0$.

Proof. Without any loss of generality we may assume that $|\psi\rangle$ is normalized and given by Eq. (18) where $a \geq b \geq c \geq 0, d>0$ and $z=x+i y$. Note that $M_{5}(\psi)=0$ if and only if $x=0$ and $4 a b c=d y^{2}$.

(i) If $|\psi\rangle$ is separable, we may assume that $a=b=c=z=0$ and $d=1$. Thus $M_{2}(\psi)=0$. Conversely, if $M_{2}(\psi)=0$ then Eq. (45) implies that $a=b=c=z=0$ and so $|\psi\rangle$ is separable.

(ii) If $|\psi\rangle$ is biseparable, we may assume that $b=c=z=0$, and so $M_{5}$ and $M_{1} M_{2}-2 M_{3}$ vanish at $|\psi\rangle$. Conversely, assume that $M_{5}$ and $M_{1} M_{2}-2 M_{3}$ vanish at $|\psi\rangle$ and that $M_{2}(\psi)>0$. Then we have $x=0$ and Eq. (59) implies that $b=c=z=0$. Now the condition $M_{2}(\psi)>0$ implies that $a d>0$, and so $|\psi\rangle$ is biseparable.

(iv) It is well known that $|\psi\rangle$ is of GHZ type if and only if $F(\psi) \neq 0$, i.e., $M_{5}(\psi)>0$.

(iii) This follows from (iv) and (ii).

Note that for the decomposability of $|\psi\rangle$ it suffices to check that $M_{2}(\psi)=0$ instead of using the Grassmann-Plücker relations.

\section{Symmetric polynomial invariants of three qubits}

The action of the local unitary group $\mathrm{U}(2) \times \mathrm{U}(2) \times \mathrm{U}(2)$ on the Hilbert space of three qubits, $\mathcal{H}=\mathbb{C}^{2} \otimes \mathbb{C}^{2} \otimes \mathbb{C}^{2}$, can be extended to the action of the semidirect product 
$G:=(\mathrm{U}(2) \times \mathrm{U}(2) \times \mathrm{U}(2)) \rtimes S_{3}$, where the symmetric group $S_{3}$ permutes the three copies of $\mathbb{C}^{2}$. We refer to the complex-valued $G$-invariant polynomial functions on $\mathcal{H}$ (viewed as a real vector space) as the symmetric polynomial invariants of three qubits. We denote by $\mathcal{B}$ the $\mathbb{C}$-algebra of the symmetric polynomial invariants.

Our first objective in this section is to compute a minimal set of generators of $\mathcal{B}$. The complex linear map $\mathcal{H} \rightarrow \wedge^{3}(V)$ which maps

$$
|i j k\rangle \mapsto e_{i+1, j+3, k+5}, \quad i, j, k \in\{0,1\}
$$

is an isometry, i.e., it preserves the norms of vectors, as well as the inner products. By using this map we shall identify $\mathcal{H}$ with the SOV subspace $W$. We shall also identify $G$ with the subgroup of $\mathrm{U}(6)$ which preserves $W$. Our second objective is to prove that the above embedding $\mathcal{H} \rightarrow \wedge^{3}(V)$ establishes a one-to-one correspondence between the $G$-orbits in $W$ and the $\mathrm{U}(6)$-orbits in $\wedge^{3}(V)$.

Let us recall some well-known facts about the algebra $\mathcal{C}$ of polynomial LU-invariants of three qubits. Let $|\psi\rangle \in \mathcal{H}$ and set $\rho=|\psi\rangle\langle\psi|$. There are six primary invariants:

$$
\begin{aligned}
Q_{1} & =\operatorname{Tr} \rho, \\
Q_{2} & =\operatorname{Tr}\left(\rho_{A}^{2}\right), \\
Q_{3} & =\operatorname{Tr}\left(\rho_{B}^{2}\right), \\
Q_{4} & =\operatorname{Tr}\left(\rho_{C}^{2}\right), \\
f_{5} & =\operatorname{Tr}\left(\rho_{A} \otimes \rho_{B} \rho_{A B}\right), \\
Q_{6} & =\mid \text { Hdet }\left.\right|^{2},
\end{aligned}
$$

where Hdet is the Cayley hyperdeterminant, see e.g., [11]. There is only one secondary invariant $f_{7}:=s_{2}^{2}$ Hdet* $^{*}$ This invariant has been constructed by M. Grassl, see [11] for the definition of $s_{2}$. For convenience, we shall replace the generators $f_{5}$ and $f_{7}$ by the invariants

$$
\begin{aligned}
& Q_{5}=\operatorname{Tr}\left(\rho_{A} \otimes \rho_{B} \rho_{A B}\right)+\operatorname{Tr}\left(\rho_{B} \otimes \rho_{C} \rho_{B C}\right)+\operatorname{Tr}\left(\rho_{A} \otimes \rho_{C} \rho_{A C}\right), \\
& Q_{7}=\Im\left(f_{7}\right) .
\end{aligned}
$$

The values of the $Q_{i}$ at $|\psi\rangle=a|100\rangle+b|010\rangle+c|001\rangle+d|111\rangle+z|000\rangle$, where $a, b, c, d$ are real and $z=x+i y$, are given by the formulae:

$$
\begin{aligned}
Q_{1}= & s_{1}+d^{2}+|z|^{2}, \\
Q_{2}= & \left(a^{2}+d^{2}\right)^{2}+\left(b^{2}+c^{2}\right)^{2}+2 s_{1}|z|^{2}+|z|^{4}, \\
Q_{3}= & \left(b^{2}+d^{2}\right)^{2}+\left(a^{2}+c^{2}\right)^{2}+2 s_{1}|z|^{2}+|z|^{4}, \\
Q_{4}= & \left(c^{2}+d^{2}\right)^{2}+\left(a^{2}+b^{2}\right)^{2}+2 s_{1}|z|^{2}+|z|^{4}, \\
Q_{5}= & 3|z|^{6}+9 s_{1}|z|^{4}+\left(9\left(a^{4}+b^{4}+c^{4}\right)+11 s_{2}+2 s_{1} d^{2}\right)|z|^{2}+6 a b c d\left(x^{2}-y^{2}\right)+ \\
& 3\left(a^{6}+b^{6}+c^{6}+d^{6}\right)+2 s_{1} d^{4}+2\left(a^{4}+b^{4}+c^{4}\right) d^{2}+3 s_{2} d^{2}+2 s_{1} s_{2}-3 s_{3}, \\
Q_{6}= & d^{2}\left(\left(4 a b c-d|z|^{2}\right)^{2}+16 a b c d x^{2}\right), \\
Q_{7}= & 8 a b c d x y \Phi(a, b, c, d, z),
\end{aligned}
$$


where $\Phi$ is given in Eq. (51). It is still true that $Q_{1}, \ldots, Q_{6}$ can be taken as the primary invariants and $Q_{7}$ as the secondary invariant. Since the transposition $(1,2) \in S_{3}$ fixes $f_{5}$, it follows that $Q_{5}$, which is the symmetrization of $f_{5}$ with respect to the cycle $(1,2,3) \in S_{3}$, is fixed by $S_{3}$. While $f_{7}$ is not fixed by $S_{3}$, one can check that $Q_{7}$ is. Moreover, a computation shows that $Q_{7}^{2} \in \mathbb{C}\left[Q_{1}, \ldots, Q_{6}\right]$. Note that now $S_{3}$ fixes the LU-invariants $Q_{1}, Q_{5}, Q_{6}, Q_{7}$, and one can check that it acts faithfully on the set $\left\{Q_{2}, Q_{3}, Q_{4}\right\}$. Thus, $S_{3}$ acts on the polynomial algebra $\mathbb{C}\left[Q_{2}, Q_{3}, Q_{4}\right]$. Since any symmetric polynomial can be expressed as a polynomial in the elementary symmetric functions of the variables, we conclude that the $S_{3}$-invariants in $\mathbb{C}\left[Q_{2}, Q_{3}, Q_{4}\right]$ are generated by

$$
Q_{2}+Q_{3}+Q_{4}, \quad Q_{2} Q_{3}+Q_{2} Q_{4}+Q_{3} Q_{4}, \quad Q_{2} Q_{3} Q_{4}
$$

We can now construct a minimal set of generators for the algebra of polynomial $G$ invariants of three qubits.

Theorem 5 The algebra $\mathcal{B}$ of polynomial $G$-invariants of three qubits is generated by the polynomials $Q_{1}, Q_{2}+Q_{3}+Q_{4}, Q_{5}, Q_{2} Q_{3}+Q_{2} Q_{4}+Q_{3} Q_{4}, Q_{6}, Q_{2} Q_{3} Q_{4}$, and $Q_{7}$ of degree 2 , $4,6,8,8,12$, and 12, respectively. The first six are the primary invariants and they generate the subalgebra $\mathcal{B}_{p}$ isomorphic to the polynomial algebra in six variables. The last generator, $Q_{7}$, is a secondary invariant. Moreover, $Q_{7}^{2} \in \mathcal{B}_{p}$ and $\mathcal{B}$ is a free module over $\mathcal{B}_{p}$ with basis $\left\{1, Q_{7}\right\}$.

Proof. Let $\mathcal{C}_{p}$ be the subalgebra of $\mathcal{C}$ generated by its primary generators $Q_{1}, \ldots, Q_{6}$. Note that $\mathcal{C}_{p}$ is invariant under the action of $S_{3}$. Since $\mathcal{C}_{p}=\mathbb{C}\left[Q_{1}, Q_{5}, Q_{6}\right] \otimes \mathbb{C}\left[Q_{2}, Q_{3}, Q_{4}\right]$ and $S_{3}$ fixes $Q_{1}, Q_{5}$, and $Q_{6}$, we deduce that the subalgebra, $\mathcal{C}_{p}^{S_{3}}$, of $S_{3}$-invariants in $\mathcal{C}_{p}$ is generated by $Q_{1}, Q_{5}, Q_{6}, Q_{2}+Q_{3}+Q_{4}, Q_{2} Q_{3}+Q_{2} Q_{4}+Q_{3} Q_{4}$, and $Q_{2} Q_{3} Q_{4}$. Thus we have $\mathcal{C}_{p}^{S_{3}}=\mathcal{B}_{p}$. Since these six polynomials are algebraically independent, $\mathcal{B}_{p}$ is isomorphic to the polynomial algebra in six variables.

Since $Q_{7}$ is fixed by $S_{3}$ and $Q_{7}^{2} \in \mathcal{C}_{p}$, we deduce that $Q_{7}^{2} \in \mathcal{C}_{p}^{S_{3}}=\mathcal{B}_{p}$. Since $\mathcal{C}$ is a free $\mathcal{C}_{p}$ module with basis $\left\{1, Q_{7}\right\}$, we can write any $f \in \mathcal{B}$ uniquely as $f=f_{1}+f_{2} Q_{7}$ with $f_{1}, f_{2} \in \mathcal{C}_{p}$. For any $\sigma \in S_{3}$ we have $f=f_{1}^{\sigma}+f_{2}^{\sigma} Q_{7}$, which implies that $f_{1}^{\sigma}=f_{1}$ and $f_{2}^{\sigma}=f_{2}$. Consequently, $f_{1}, f_{2} \in \mathcal{B}_{p}$. We conclude that $f \in \mathcal{B}_{p}\left[Q_{7}\right]$, and so $\mathcal{B}=\mathcal{B}_{p}\left[Q_{7}\right]$. As in the proof of Theorem 2 , we can show that $\mathcal{B}$ is a free $\mathcal{B}_{p}$ module with basis $\left\{1, Q_{7}\right\}$.

We can now show that the embedding (60) gives a one-to-one correspondence between $G$ equivalence classes of pure three-qubit states in $\mathcal{H}$ and the LU-equivalence classes of pure fermionic states in $\wedge^{3}(V)$.

Theorem 6 The restriction map $\left.f \rightarrow f\right|_{W}$ from the algebra $\mathcal{A}:=\mathcal{P}_{\mathbb{R}}^{\mathrm{U}}$ to the $\mathbb{C}$-algebra of polynomial functions on $W$, viewed as a real vector space, is injective and its image is the algebra $\mathcal{B}$ of polynomial $G$-invariants of three qubits. In particular these two algebras are isomorphic as graded algebras. If $\mathcal{O}$ is an $\mathrm{U}(6)$-orbit in $\wedge^{3}(V)$ then $\mathcal{O} \cap W$ is a single $G$ orbit in $W$, and the map which sends $\mathcal{O}$ to $\mathcal{O} \cap W$ is a one-to-one correspondence between the $L U$-equivalence classes of pure fermionic states in $\wedge^{3}(V)$ and the $G$-equivalence classes of pure 3-qubit states in $W$. 
Proof. For $f \in \mathcal{A}$ we denote by $f_{W}$ the restriction of $f$ to the subspace $W \subseteq \wedge^{3}(V)$. Let us also denote by $\mathcal{A}_{W}$ the image of $\mathcal{A}$ by this restriction map. Recall that each pure fermionic state is LU-equivalent to an SOV. This implies that if $f_{W}=0$ and $f \in \mathcal{A}$, then $f=0$. Thus, the restriction map $\mathcal{A} \rightarrow \mathcal{A}_{W}$ is an isomorphism of graded algebras. As $G$ is a subgroup of $\mathrm{U}(6)$, it is easy to verify that for $f \in \mathcal{A}$ we have $f_{W} \in \mathcal{B}$. Hence, $\mathcal{A}_{W}$ is a subalgebra of $\mathcal{B}$. It follows from Theorem 5 that the algebras $\mathcal{A}_{W}$ and $\mathcal{B}$ have the same Poincaré series, namely the one given by Eq. (31). As $\mathcal{A}_{W} \subseteq \mathcal{B}$, we must have the equality $\mathcal{A}_{W}=\mathcal{B}$.

Now let $\mathcal{O}$ be an $\mathrm{U}(6)$-orbit in $\wedge^{3}(V)$. As mentioned above, we must have $\mathcal{O} \cap W \neq \emptyset$. Since $\mathcal{A}_{W}=\mathcal{B}$, it follows that $\mathcal{O} \cap W$ is a single $G$-orbit. Hence, the map which sends $\mathcal{O} \rightarrow \mathcal{O} \cap W$ is indeed the one-to-one correspondence as asserted in the theorem.

Corollary 7 A polynomial $f \in \mathcal{C}$ is invariant under qubit permutations (i.e., it belongs to the subalgebra $\mathcal{B})$ if and only if its restriction $f^{\prime}:=\left.f\right|_{W_{6}}$ is a symmetric polynomial with respect to the variables $a, b, c$.

Proof. Recall that $W_{6}$ is an $S_{3}$-invariant real subspace of $W$ of dimension six, with coordinates $a, b, c, d, x, y$. It is easy to verify that, when restricted to $W_{6}, S_{3}$ fixes the coordinates $d, x, y$ and permutes the coordinates $a, b, c$. Now the assertion follows from the fact that the map which sends $f \in \mathcal{C}$ to its restriction $f^{\prime}$ is injective.

The one-to-one correspondence mentioned in Theorem $[$ is in fact a homeomorphism between the orbit spaces $\wedge^{3}(V) / \mathrm{U}(6)$ and $W / G$. As sets, these orbit spaces are just the set of $\mathrm{U}(6)$-orbits in $\wedge^{3}(V)$ and the set of $G$-orbits in $W$, respectively. However, they are also toplogical spaces with respect to the quotient topology arising from the projection maps $\pi_{V}: \wedge^{3}(V) \rightarrow \wedge^{3}(V) / \mathrm{U}(6)$ and $\pi_{W}: W \rightarrow W / G$. For additional properties of orbit spaces of compact Lie groups we refer the reader to [40, Chapter I].

Corollary 8 The one-to-one correspondence $\wedge^{3}(V) / \mathrm{U}(6) \rightarrow W / G$, constructed in Theorem [6] is a homeomorphism.

Proof. Let us denote by $T$ the inverse of this correspondence. Thus, for any $|\phi\rangle \in W$ we have $T(G \cdot|\phi\rangle)=\mathrm{U}(6) \cdot|\phi\rangle$. Since the composite of $\pi_{V}$ and the inclusion map $\iota: W \rightarrow \wedge^{3}(V)$ is continuous and coincides with $T \circ \pi_{W}$, we deduce that $T$ is continuous. It remains to show that $T^{-1}$ is continuous. By Lemma 1 we know that $\mathrm{U}(6) \cdot W=\wedge^{3}(V)$. Let $\mathrm{U}(6)$ act trivially on $W / G$. If $|\alpha\rangle,|\beta\rangle \in W$ are LU-equivalent, then by Theorem 6 they belong to the same $G$-orbit and so $\pi_{W}(\alpha)=\pi_{W}(\beta)$. Therefore we can apply [40, Theorem 3.3] to the $\mathrm{U}(6)$-spaces $\wedge^{3}(V) / \mathrm{U}(6)$ and $W / G$. We conclude that $\pi_{W}$ extends uniquely to a continuous map $\pi_{W}^{\prime}: \wedge^{3}(V) \rightarrow W / G$ which is constant on $\mathrm{U}(6)$-orbits. Since $\pi_{V} \circ \iota=T \circ \pi_{W}=T \circ \pi_{W}^{\prime} \circ \iota$, it follows easily that $\pi_{V}=T \circ \pi_{W}^{\prime}$. Hence, $\pi_{W}^{\prime}=T^{-1} \circ \pi_{V}$ and so $T^{-1}$ must be continuos.

\section{The canonical region}

In this section we shall work with normalized states only, which means that we have $M_{1}=1$. For convenience, we shall identify any point $(a, b, c, d, z) \in \mathbb{R}^{4} \times \mathbb{C}$ with the corresponding 
vector $|\psi\rangle$ given by Eq. (18). We shall always write the complex coordinate $z$ as $z=x+i y$, where $x$ and $y$ are real. Thus we have identified the subspace $W_{6}$ with the product $\mathbb{R}^{4} \times \mathbb{C}$. We denote by $\Sigma$ the unit sphere of $W_{6}$, i.e., the set of all points $(a, b, c, d, z) \in W_{6}$ such that $a^{2}+b^{2}+c^{2}+d^{2}+|z|^{2}=1$. We shall denote by $\Sigma_{i}, i=1,2,3$, the unit sphere of the subspace $V_{i}$ of $V$, see Eq. (2).

\subsection{The canonical region $\Delta$}

To begin, we introduce two continuous functions $\mu: \wedge^{3}(V) \rightarrow \mathbb{R}$ and $\mu^{\prime}: W \rightarrow \mathbb{R}$.

Definition 9 For $|\psi\rangle \in \wedge^{3}(V)$, we set

$$
\mu(\psi)=\max _{\alpha, \beta, \gamma}|\langle\alpha \wedge \beta \wedge \gamma \mid \psi\rangle|, \quad\|\alpha \wedge \beta \wedge \gamma\|=1
$$

and for $|\psi\rangle \in W$, we set

$$
\mu^{\prime}(\psi)=\max _{\alpha, \beta, \gamma}|\langle\alpha \wedge \beta \wedge \gamma \mid \psi\rangle|, \quad(\alpha, \beta, \gamma) \in \Sigma_{1} \times \Sigma_{2} \times \Sigma_{3}
$$

We prove that $\mu^{\prime}$ is the restriction of $\mu$.

Lemma 10 For $|\psi\rangle \in W$, we have $\mu^{\prime}(\psi)=\mu(\psi)$.

Proof. Note that $\mu^{\prime}(\psi) \leq \mu(\psi)$. Let $|\alpha \wedge \beta \wedge \gamma\rangle$ be a unit vector such that $\mu(\psi)=$ $|\langle\alpha \wedge \beta \wedge \gamma \mid \psi\rangle|$. Let $L=\operatorname{span}\{\alpha, \beta, \gamma\}$ and choose unit vectors $\left|\alpha^{\prime}\right\rangle,\left|\beta^{\prime}\right\rangle,\left|\gamma^{\prime}\right\rangle \in L$ such that $\left|\alpha^{\prime}\right\rangle \in V_{2}+V_{3},\left|\beta^{\prime}\right\rangle \in V_{1}+V_{3}$, and $\left|\gamma^{\prime}\right\rangle \in V_{1}+V_{2}$. At least two of these three vectors must be linearly independent, say $\left|\alpha^{\prime}\right\rangle$ and $\left|\gamma^{\prime}\right\rangle$. We can write $\left|\alpha^{\prime}\right\rangle=c_{2}\left|\gamma_{2}\right\rangle+c_{3}\left|\gamma_{3}\right\rangle$ where $c_{2}, c_{3} \geq 0$, and $\left|\gamma_{2}\right\rangle \in V_{2}$ and $\left|\gamma_{3}\right\rangle \in V_{3}$ are unit vectors. Similarly, we write $\left|\gamma^{\prime}\right\rangle=a_{1}\left|\alpha_{1}\right\rangle+a_{2}\left|\alpha_{2}\right\rangle$ where $a_{1}, a_{2} \geq 0$, and $\left|\alpha_{1}\right\rangle \in V_{1}$ and $\left|\alpha_{2}\right\rangle \in V_{2}$ are unit vectors. As $\mu(\psi)>0$, either $a_{1}$ or $c_{3}$ is non-zero, and so we have $a_{2} c_{2}<1$. We have $\left|\left\langle\alpha^{\prime} \mid \gamma^{\prime}\right\rangle\right|^{2}=t a_{2}^{2} c_{2}^{2}$ where $t:=\left|\left\langle\alpha_{2} \mid \gamma_{2}\right\rangle\right|^{2} \leq 1$. Finally, let $\left|\beta^{\prime \prime}\right\rangle=b_{1}\left|\beta_{1}\right\rangle+b_{2}\left|\beta_{2}\right\rangle+b_{3}\left|\beta_{3}\right\rangle \in L$, with $\left|\beta_{i}\right\rangle \in V_{i}$ and $b_{i} \geq 0$, be a nonzero vector orthogonal to $\left|\alpha^{\prime}\right\rangle$ and $\left|\gamma^{\prime}\right\rangle$. We fix its norm to be $\left\|\beta^{\prime \prime}\right\|=1 / \sqrt{1-t a_{2}^{2} c_{2}^{2}}$. Since both $|\alpha \wedge \beta \wedge \gamma\rangle$ and $\left|\gamma^{\prime} \wedge \beta^{\prime \prime} \wedge \alpha^{\prime}\right\rangle$ are unit vectors in $\wedge^{3}(L)$, they differ only by a phase factor. Hence, we can assume that $|\alpha\rangle=\left|\gamma^{\prime}\right\rangle,|\beta\rangle=\left|\beta^{\prime \prime}\right\rangle$ and $|\gamma\rangle=\left|\alpha^{\prime}\right\rangle$. Since

$$
\begin{aligned}
|\langle\alpha \wedge \beta \wedge \gamma \mid \psi\rangle| \leq & a_{1} b_{2} c_{3}\left|\left\langle\alpha_{1} \wedge \beta_{2} \wedge \gamma_{3} \mid \psi\right\rangle\right| \\
& +a_{1} b_{3} c_{2}\left|\left\langle\alpha_{1} \wedge \beta_{3} \wedge \gamma_{2} \mid \psi\right\rangle\right|+a_{2} b_{1} c_{3}\left|\left\langle\alpha_{2} \wedge \beta_{1} \wedge \gamma_{3} \mid \psi\right\rangle\right|
\end{aligned}
$$

we infer that $\mu(\psi) \leq\left(a_{1} b_{2} c_{3}+a_{1} b_{3} c_{2}+a_{2} b_{1} c_{3}\right) \mu^{\prime}(\psi)$. We have $\mu(\psi) \leq \mu^{\prime}(\psi)$ because

$$
\begin{aligned}
\left(a_{1} b_{2} c_{3}+a_{1} b_{3} c_{2}+a_{2} b_{1} c_{3}\right)^{2} & \leq\left(a_{1}^{2} c_{3}^{2}+a_{1}^{2} c_{2}^{2}+a_{2}^{2} c_{3}^{2}\right)\left(b_{1}^{2}+b_{2}^{2}+b_{3}^{2}\right) \\
& =\frac{1-a_{2}^{2} c_{2}^{2}}{1-t a_{2}^{2} c_{2}^{2}} \leq 1
\end{aligned}
$$

Consequently, $\mu^{\prime}(\psi)=\mu(\psi)$. 
For $|\psi\rangle \in W_{6}$ given by Eq. (18), we have

$$
\begin{aligned}
\mu(\psi)^{2} & =\max _{u_{1}, u_{2}, u_{3}} \mid\left\langle\left.\psi\left|\left(\xi_{1}|1\rangle+\eta_{1}|2\rangle\right) \wedge\left(\xi_{2}|3\rangle+\eta_{2}|4\rangle\right) \wedge\left(\xi_{3}|5\rangle+\eta_{3}|6\rangle\right)\right\rangle\right|^{2}\right. \\
& =\max _{u_{1}, u_{2}, u_{3}}\left|a \eta_{1} \xi_{2} \xi_{3}+b \xi_{1} \eta_{2} \xi_{3}+c \xi_{1} \xi_{2} \eta_{3}+d \eta_{1} \eta_{2} \eta_{3}+z^{*} \xi_{1} \xi_{2} \xi_{3}\right|^{2},
\end{aligned}
$$

where the maximum is taken over all unit vectors $u_{i}=\left(\xi_{i}, \eta_{i}\right) \in \mathbb{C}^{2}, i=1,2,3$.

Definition 11 We denote by $\Delta$ the subset of $\Sigma$ consisting of all points $p=(a, b, c, d, z)$, $z=x+i y$, such that (i) $a \geq b \geq c \geq 0, x \geq 0$ and (ii) $d=\mu(\psi)$, where the state $|\psi\rangle$ corresponds to $p$.

Since $\mu$ is a continuous function, it follows that $\Delta$ is a closed (and so compact) subset of $\Sigma$. The relative interior of $\Delta$ as a subset of $\Sigma$, is the set $\Delta^{0}$ of all points $p \in \Delta$ for which there exists $\varepsilon>0$ such that $q \in \Sigma$ and $\|q-p\|<\varepsilon$ imply that $q \in \Delta$. As $\Delta$ is closed, the relative boundary $\partial \Delta$ of $\Delta$ is the set-theoretic difference $\Delta \backslash \Delta^{0}$. At this point we do not know whether $\Delta^{0} \neq \emptyset$, but we shall see later that this is the case.

Note that $\Delta$ is contained in the northern hemisphere of $\Sigma$ defined by the inequality $d>0$. We shall prove now that $\Delta$ and $\Delta^{0}$ are connected. For convenience, we shall identify in this lemma the points $p \in \mathbb{R}^{4} \times \mathbb{C}$ with the corresponding vectors $0 p$.

Lemma 12 Let $p, q \in \Delta$ and let $r=t p+(1-t) q$ where $0<t<1$. Then the point $s=r /\|r\|$ belongs to $\Delta$. Consequently, $\Delta$ and $\Delta^{0}$ are connected.

Proof. $\quad$ Since $p$ and $q$ satisfy the linear inequalities (i) of Definition 11, so do $r$ and $s$. It remains to prove that $s$ also satisfies the condition (ii) of that definition. Let $\left|\psi_{1}\right\rangle$, $\left|\psi_{2}\right\rangle$, and $\left|\psi_{3}\right\rangle$ be the states corresponding to the points $p, q$, and $s$, respectively, and note that $\|r\| \cdot\left|\psi_{3}\right\rangle=t\left|\psi_{1}\right\rangle+(1-t)\left|\psi_{2}\right\rangle$. Let $p=\left(a_{1}, b_{1}, c_{1}, d_{1}, z_{1}\right), q=\left(a_{2}, b_{2}, c_{2}, d_{2}, z_{2}\right)$, and $s:=\left(a_{3}, b_{3}, c_{3}, d_{3}, z_{3}\right)$. By definition of $\mu$ we have

$$
\begin{aligned}
\mu(s) & =\max _{\alpha, \beta, \gamma}\left|\left\langle\alpha \wedge \beta \wedge \gamma \mid \psi_{3}\right\rangle\right| \\
& \leq \frac{1}{\|r\|}\left(t \cdot \max _{\alpha, \beta, \gamma}\left|\left\langle\alpha \wedge \beta \wedge \gamma \mid \psi_{1}\right\rangle\right|+(1-t) \cdot \max _{\alpha, \beta, \gamma}\left|\left\langle\alpha \wedge \beta \wedge \gamma \mid \psi_{2}\right\rangle\right|\right) \\
& =\frac{1}{\|r\|}\left(t d_{1}+(1-t) d_{2}\right)=d_{3},
\end{aligned}
$$

where the maxima are over all decomposable 3-vectors $|\alpha \wedge \beta \wedge \gamma\rangle$ of unit norm. As the inequality $\mu(s) \geq d_{3}$ is trivial, the proof is completed.

In the following lemma we prove a basic property of $\Delta$.

Lemma 13 Each LU-orbit of normalized fermionic states $|\psi\rangle \in \wedge^{3}(V)$ has a representative in $\Delta$. 
Proof. Let $|\psi\rangle \in \wedge^{3}(V)$ be a unit vector. By Lemma 1 the LU-orbit of $|\psi\rangle$ meets $W$, and so we may assume that $|\psi\rangle \in W$, where $W=V_{1} \wedge V_{2} \wedge V_{3}$ is the SOV subspace (see Eq. (2) ). By Lemma 10 there exists unit vectors $|\alpha\rangle \in V_{1},|\beta\rangle \in V_{2}$, and $|\gamma\rangle \in V_{3}$ such that $\mu(\psi)=\langle\alpha \wedge \beta \wedge \gamma \mid \psi\rangle$. Let $g \in \mathrm{U}(2) \times \mathrm{U}(2) \times \mathrm{U}(2)$ be chosen so that $g|\alpha\rangle=|2\rangle, g|\beta\rangle=|4\rangle$, and $g|\gamma\rangle=|6\rangle$. By replacing $|\psi\rangle$ with $g \cdot|\psi\rangle$, we obtain that $\mu:=\mu(\psi)=\left\langle e_{246} \mid \psi\right\rangle$. Thus we can write $|\psi\rangle=\sum_{i, j, k \in\{0,1\}} t_{i j k} e_{i j k}$, where $t_{i j k} \in \mathbb{C}$, with $t_{111}=\mu$.

It is now easy to show that $t_{i j k}=0$ if exactly one of $i, j, k$ is zero. Assume that $i=0$ and $j=k=1$. Let $|\varphi\rangle=|\alpha \wedge \beta \wedge \gamma\rangle$ where $\alpha=\left(t_{011}|1\rangle+\mu|2\rangle\right) / \sqrt{\left|t_{011}\right|^{2}+\mu^{2}}, \beta=|4\rangle$,

and $\gamma=|6\rangle$. Then we have $\mu \geq|\langle\varphi \mid \psi\rangle|=\sqrt{\left|t_{011}\right|^{2}+\mu^{2}}$, and so $t_{011}=0$. Consequently $|\psi\rangle \in W_{6}$.

By applying a suitable diagonal LU transformation, we can further assume that $t_{i j k} \geq 0$ if exactly two of $i, j, k$ are zero. By using the action of the symmetric group $S_{3}$ described in Sec. 3, we can further assume that $t_{100} \geq t_{010} \geq t_{001} \geq 0$. Finally, the diagonal LU transformation, which fixes the basis vectors $|i\rangle$ for $i=2,4,6$ and multiplies them with -1 for $i=1,3,5$, will replace $t_{000}$ with $-t_{000}$ and will not change any other $t_{i j k}$. This means that we can assume that also $\Re\left(t_{000}\right) \geq 0$. Hence $|\psi\rangle \in \Delta$.

In view of this lemma, we shall refer to $\Delta$ as the canonical region. Evidently, two LUequivalent states in $\Delta$ have the same coefficient $d$. Thus

Corollary 14 Two points in $\Delta$ having different d-coordinate are not LU-equivalent.

\subsection{Inequalities defining $\Delta$}

Our definition of $\Delta$ is not easy to use because the condition (ii) is hard to verify. We shall prove that this condition can be replaced by the inequality $d>0$ and four additional inequalities. The set of inequalities defining $\Delta$ as a subset of $\Sigma$ will be simplified later, see Proposition 23. We need an auxiliary lemma, but first let us make a couple of observations.

If $\alpha, \beta, \gamma, \delta$ are arbitrary complex numbers, then the following identity holds

$$
\left(|\alpha|^{2}+|\beta|^{2}+|\gamma|^{2}+|\delta|^{2}\right)^{2}-4|\alpha \delta-\beta \gamma|^{2}=\left(|\alpha|^{2}-|\beta|^{2}+|\gamma|^{2}-|\delta|^{2}\right)^{2}+4\left|\alpha \beta^{*}+\gamma \delta^{*}\right|^{2} .
$$

By the Cauchy-Schwarz inequality we have

$$
\max _{u=(\xi, \eta)}|\alpha \xi+\beta \eta|^{2}=|\alpha|^{2}+|\beta|^{2},
$$

where $\alpha$ and $\beta$ are arbitrary complex numbers, and the maximum is taken over all unit vectors $u=(\xi, \eta) \in \mathbb{C}^{2}$. This observation can be generalized as follows.

Lemma 15 Let $\alpha, \beta, \gamma, \delta$ be any complex numbers. Denote the maximum of $\mid\left(\alpha \xi_{1}+\beta \eta_{1}\right) \xi_{2}+$ $\left.\left(\gamma \xi_{1}+\delta \eta_{1}\right) \eta_{2}\right|^{2}$ taken over all unit vectors $u_{i}=\left(\xi_{i}, \eta_{i}\right) \in \mathbb{C}^{2}, i=1,2$, by $\lambda$. Then

$$
\lambda=\frac{1}{2}\left(|\alpha|^{2}+|\beta|^{2}+|\gamma|^{2}+|\delta|^{2}+\sqrt{\left(|\alpha|^{2}+|\beta|^{2}+|\gamma|^{2}+|\delta|^{2}\right)^{2}-4|\alpha \delta-\beta \gamma|^{2}}\right) .
$$


Proof. By Eq. (84) we have

$$
\lambda=\max _{u_{1}}\left(\left|\alpha \xi_{1}+\beta \eta_{1}\right|^{2}+\left|\gamma \xi_{1}+\delta \eta_{1}\right|^{2}\right) .
$$

The function that we are maximizing here can be rewritten as

$$
\begin{aligned}
& \frac{1}{2}\left(|\alpha|^{2}+|\beta|^{2}+|\gamma|^{2}+|\delta|^{2}\right)+\frac{1}{2}\left(|\alpha|^{2}-|\beta|^{2}+|\gamma|^{2}-|\delta|^{2}\right)\left(\left|\xi_{1}\right|^{2}-\left|\eta_{1}\right|^{2}\right) \\
& \quad+2 \Re\left(\left(\alpha \beta^{*}+\gamma \delta^{*}\right) \xi_{1} \eta_{1}^{*}\right) .
\end{aligned}
$$

Since $\left(\left|\xi_{1}\right|^{2}-\left|\eta_{1}\right|^{2}, 2 \xi_{1} \eta_{1}^{*}\right)$ runs through all unit vectors in $\mathbb{C}^{2}$ (up to an overall phase factor), another application of Eq. (84) gives

$$
\lambda=\frac{1}{2}\left(|\alpha|^{2}+|\beta|^{2}+|\gamma|^{2}+|\delta|^{2}+\sqrt{\left(|\alpha|^{2}-|\beta|^{2}+|\gamma|^{2}-|\delta|^{2}\right)^{2}+4\left|\alpha \beta^{*}+\gamma \delta^{*}\right|^{2}}\right) .
$$

By using the identity (83), this formula can be rewritten in the form (85).

Note that $a^{2}+b^{2}+c^{2}+d^{2}+|z|^{2}=1$ implies the equality

$$
\left(d^{2}-a^{2}\right)\left(d^{2}-b^{2}\right)-d^{2}|z|^{2}=a^{2} b^{2}+c^{2} d^{2}+d^{2}\left(2 d^{2}-1\right)
$$

which will be tacitly used in the next proof.

Theorem 16 Let $p=(a, b, c, d, z) \in \Sigma, z=x+i y$, such that $a \geq b \geq c \geq 0, x \geq 0$, and let $|\psi\rangle$ be the corresponding normalized pure fermionic state.

(a) If $p \in \Delta$, then $d>0$ and the following inequalities hold

$$
\begin{aligned}
& a^{2} b^{2}+c^{2} d^{2}+d^{2}\left(2 d^{2}-1\right) \geq 0 \\
& d\left(d^{2}-s_{1}\right)-2 a b c \geq 0 \\
& 2\left(a^{2} b^{2}+c^{2} d^{2}+d^{2}\left(2 d^{2}-1\right)\right)\left(d^{2}-s_{1}\right)-x^{2}(a b+c d)^{2}-y^{2}(a b-c d)^{2} \geq 0 \\
& d^{2}\left(2 d^{2}-1\right)\left(d^{2}-s_{1}\right)-2 a b c d\left(x^{2}-y^{2}\right)-4 s_{3} \geq 0
\end{aligned}
$$

If equality holds in (90), then $b x=(a b-c d) y=0$. If equality holds in (91) or (92), then $b x=0$.

(b) Conversely, if $d>0$ and the inequalities (90)-(93) hold, then $p \in \Delta$.

Proof. To simplify notation, we shall denote by $\alpha, \beta, \gamma, \delta$ the left hand sides of (90)-(93), respectively. By applying Lemma 15 to compute the maximum in Eq. (81) over unit vectors $u_{1}$ and $u_{2}$ only, we obtain that

$$
\mu(\psi)^{2}=\frac{1}{2} \max _{u_{3}}\left(P+\sqrt{P^{2}-4|Q|^{2}}\right)
$$

where $P=\left|z^{*} \xi_{3}+c \eta_{3}\right|^{2}+\left(a^{2}+b^{2}\right)\left|\xi_{3}\right|^{2}+d^{2}\left|\eta_{3}\right|^{2}, Q=a b \xi_{3}^{2}-d z^{*} \xi_{3} \eta_{3}-c d \eta_{3}^{2}$, and $u_{3}=$ $\left(\xi_{3}, \eta_{3}\right) \in \mathbb{C}^{2}$ is any unit vector. 
Proof of (a). As $p \in \Delta$, we have $d=\mu(\psi)>0$. Consequently, the inequality

$$
2 d^{2} \geq P+\sqrt{P^{2}-4|Q|^{2}}
$$

is valid for all unit vectors $u_{3}=\left(\xi_{3}, \eta_{3}\right) \in \mathbb{C}^{2}$. We now assume that $\eta_{3} \neq 0$ and set $\xi_{3} / \eta_{3}=t e^{i \theta}$ where $t$ and $\theta$ are real numbers. By moving the term $P$ in (95) to the left hand side and squaring both sides, we obtain that

$$
d^{4}-d^{2}\left(\left|t z^{*} e^{i \theta}+c\right|^{2}+\left(a^{2}+b^{2}\right) t^{2}+d^{2}\right)\left|\eta_{3}\right|^{2}+\left|a b t^{2} e^{2 i \theta}-d z^{*} t e^{i \theta}-c d\right|^{2} \cdot\left|\eta_{3}\right|^{4} \geq 0 .
$$

We divide the left hand side by $\left|\eta_{3}\right|^{4}$ and use the fact that $\left|\eta_{3}\right|^{-2}=1+t^{2}$. Then the left hand side is divisible by $t^{2}$, and by omitting this factor we obtain that

$$
\alpha t^{2}-2 d \Re\left(\left(a b z+c d z^{*}\right) e^{i \theta}\right) t+d\left(d\left(d^{2}-s_{1}\right)-2 a b c \cos 2 \theta\right) \geq 0 .
$$

Since this inequality holds for all real $t$ and $\theta$, we deduce that $\alpha \geq 0$ and $\beta \geq 0$, i.e., the inequalities (90) and (91) hold. As the discriminant of the quadratic polynomial in $t$ on the left hand side of (97) must be nonpositive for all real $\theta$, we have

$$
\alpha\left(d\left(d^{2}-s_{1}\right)-2 a b c \cos 2 \theta\right)-d\left(\Re\left(\left(a b z+c d z^{*}\right) e^{i \theta}\right)\right)^{2} \geq 0 .
$$

If $\alpha=0$, then we must have $a b z+c d z^{*}=0$, which is equivalent to $b x=(a b-c d) y=0$. Now assume that $\beta=0$. By setting $\theta=0$ in (98), we deduce that $\Re\left(a b z+c d z^{*}\right)=0$, i.e., $b x=0$.

Next we shall prove (92) and (93). We can rewrite the inequality (98) as

$$
d \gamma \geq\left(4 \alpha a b c+d x^{2}(a b+c d)^{2}-d y^{2}(a b-c d)^{2}\right) \cos 2 \theta-2 d x y\left(a^{2} b^{2}-c^{2} d^{2}\right) \sin 2 \theta .
$$

Since this inequality holds for all real $\theta$, it follows that $\gamma \geq 0$, i.e., the inequality (92) holds. Moreover, we must have

$$
d^{2} \gamma^{2}-\left(4 \alpha a b c+d x^{2}(a b+c d)^{2}-d y^{2}(a b-c d)^{2}\right)^{2}-\left(2 d x y\left(a^{2} b^{2}-c^{2} d^{2}\right)\right)^{2} \geq 0 .
$$

If $\gamma=0$, then the two equalities $x y\left(a^{2} b^{2}-c^{2} d^{2}\right)=0$ and $4 \alpha a b c+d x^{2}(a b+c d)^{2}-d y^{2}(a b-$ $c d)^{2}=0$ must hold. If also $x>0$ then from the first equality we deduce that $y(a b-c d)=0$, and then from the second one we deduce that $b=0$. Thus $\gamma=0$ implies that $b x=0$.

The left hand side of (100) factorizes and we obtain that

$$
4\left(d^{2}-a^{2}\right)\left(d^{2}-b^{2}\right) \alpha \delta \geq 0 .
$$

Assume that $\delta<0$. Then $d=a$ or $\alpha=0$. If $d=a$, then $\beta \geq 0$ implies that $b=c=0$ and $d^{2}-s_{1}=0$, which contradicts the assumption that $\delta<0$. Hence, we must have $d>a$ and $\alpha=0$. It follows that $b x=(a b-c d) y=0$. If $b=0$, then $\delta=d^{2}\left(2 d^{2}-1\right)\left(d^{2}-a^{2}\right)<0$ contradicts the inequality (90). Hence $b>0$, and so $x=0$ and $\left(d^{2}-a^{2}\right)\left(d^{2}-b^{2}\right)=d^{2} y^{2}$. As $d>a$, this implies that $y \neq 0$ and so $a b=c d$ and $c>0$. Now (90) implies that $c^{2}+d^{2}=1 / 2$. The inequality $\delta<0$ becomes $-2 c^{2} d^{2}\left(d^{2}-s_{1}\right)+2 c^{2} d^{2} y^{2}-4 c^{4} d^{2}<0$. After canceling the 
factor $2 c^{2} d^{2}$, we obtain that $a^{2}+b^{2}+y^{2}<1 / 2$ which gives a contradiction. Hence, we must have $\delta \geq 0$.

Proof of (b). We have to prove that $d=\mu(\psi)$. It is immediate from the definition of $\mu(\psi)$ that $d \leq \mu(\psi)$. In order to prove that $d \geq \mu(\psi)$, we will reverse the main steps in the proof of (a).

Since $d>0$, it follows from $\beta \geq 0$ that $d^{2} \geq s_{1}$ and, in particular, $d \geq a$. Since $\alpha \geq 0$ and $\delta \geq 0$, the inequality (101) holds, and so does the inequality (100). As $\gamma \geq 0$, we deduce that the inequality (99) holds for all real $\theta$, and so does (98). Since $\alpha \geq 0$ and $\beta \geq 0$, this implies that the inequality (97) holds for all real $t$ and $\theta$, and that the inequality (96) holds for all unit vectors $u_{3}=\left(\xi_{3}, \eta_{3}\right) \in \mathbb{C}^{2}$ with $\xi_{3}=t e^{i \theta} \eta_{3}$. We can rewrite the inequality (96) as $\left(2 d^{2}-P\right)^{2} \geq P^{2}-4|Q|^{2}$. By using the identity (83), one can easily show that $P^{2}-4|Q|^{2} \geq 0$.

We claim that $2 d^{2}-P \geq 0$ for all unit vectors $u_{3}$. By computing the maximum of $P$ over all $u_{3}$, our claim asserts that $2 d^{2} \geq 1 / 2+\left(\left(1 / 2-c^{2}-d^{2}\right)^{2}+c^{2}|z|^{2}\right)^{1 / 2}$. Note that (90) implies that $d^{2} \geq a^{2}+|z|^{2}$. As $2 d^{2} \geq b^{2}+c^{2}$, we obtain that $4 d^{2} \geq 1$, i.e., $2 d^{2}-1 / 2 \geq 0$. Hence, our claim is equivalent to the inequality $\left(2 d^{2}-1 / 2\right)^{2} \geq\left(1 / 2-c^{2}-d^{2}\right)^{2}+c^{2}|z|^{2}$. This can be simplified to $d^{2}\left(3 d^{2}-1\right) \geq\left(d^{2}-a^{2}-b^{2}\right) c^{2}$. Therefore it suffices to prove that $3 d^{2}-1 \geq c^{2}$. In fact the stronger inequality $3 d^{2}-1 \geq a^{2}$ holds. Indeed, the inequality (91) implies that $d^{2} \geq a^{2}+b^{2}+c^{2}$. By adding this inequality and the inequality $d^{2} \geq a^{2}+|z|^{2}$, we obtain that $2 d^{2} \geq 1+a^{2}-d^{2}$. This completes the proof of our claim.

By extracting square roots on both sides of $\left(2 d^{2}-P\right)^{2} \geq P^{2}-4|Q|^{2}$, we conclude that the inequality (95) is valid for all unit vectors $u_{3}$. By invoking Eq. (94), we obtain that $d \geq \mu(\psi)$. This completes the proof of part (b), and of the theorem.

We derive two consequences of the above theorem.

\section{Corollary 17}

(i) $\Delta$ is the closure of $\Delta^{0}$ (in particular, $\Delta^{0} \neq \emptyset$ ).

(ii) All points $p=(a, b, c, d, z) \in \Delta$ satisfy the inequality

$$
2 a b c+d\left(2 d^{2}-1\right) \geq 0 .
$$

Proof. (i) It follows from Theorem 16 that $\Delta^{0} \neq \emptyset$. For instance, all inequalities defining $\Delta$ are strict at the point $p=(8,4,2,11,2+4 i) / 15$ and so $p \in \Delta^{0}$. Now, the assertion follows from Lemma 12 ,

(ii) In view of (i), it suffices to prove this inequality when $p \in \Delta^{0}$. If $\delta$ denotes the left hand side of Eq. (93), then $\delta+4 a b c d x^{2}$ is a polynomial in $a, b, c, d$ and $|z|^{2}$. After substituting $|z|^{2}=$ $1-s_{1}-d^{2}$ in this polynomial, we obtain the inequality $\left(d\left(d^{2}-s_{1}\right)-2 a b c\right)\left(2 a b c+d\left(2 d^{2}-1\right)\right) \geq 0$. As $p \in \Delta^{0}$ we have $d\left(d^{2}-s_{1}\right)-2 a b c>0$ and so $2 a b c+d\left(2 d^{2}-1\right) \geq 0$.

\subsection{The boundary of $\Delta$}

We can now describe the boundary of $\Delta$. 
Proposition 18 For $p=(a, b, c, d, z) \in \Delta$ with $z=x+i y$, we have $p \in \partial \Delta$ if and only if $c x(a-b)(b-c) \Phi(p)=0$.

Proof. Assume that $c x(a-b)(b-c) \Phi(p)=0$. If $c x(a-b)(b-c)=0$, it is obvious that $p \in \partial \Delta$. Now let $c x(a-b)(b-c) \neq 0$, i.e., $a>b>c>0$ and $x>0$. Then we have $\Phi(p)=0$. By computing the gradient of $\Phi$, in the 6 -dimensional Euclidean space with coordinates $a, b, c, d, x, y$, we find that at the point $p$ we have $a \frac{\partial \Phi}{\partial b}-b \frac{\partial \Phi}{\partial a}=-2 c\left(a^{2}-b^{2}\right)\left(4 a b c+d\left(x^{2}-y^{2}\right)\right)$ and $x \frac{\partial \Phi}{\partial y}-y \frac{\partial \Phi}{\partial x}=8 a b c d x y$. Since at least one of these two expressions is nonzero, the gradient $(\nabla \Phi)_{p}$ is not parallel to $p$. Consequently, in any neighborhood of $p$ on the unit sphere of $W_{6}$ the polynomial $\Phi$ takes both positive and negative values. Hence $p \in \partial \Delta$ by Theorem 16 .

We shall prove the converse by contradiction. Thus, in addition to the hypothesis $p \in \partial \Delta$, we shall assume that $c x(a-b)(b-c) \Phi(p) \neq 0$. Consequently, we have $a>b>c>0, x>0$. As $d>0$ and $p \in \partial \Delta$, we infer that equality must hold in at least one of the inequalities (90), (91), (92). But in each of these three cases, part (a) of Theorem 16 asserts that equality implies that $b x=0$. Hence, we have a contradiction.

To demonstrate that the hypothesis $p \in \Delta$ cannot be omitted, consider the point $p=$ $(12,6,4,9,2,2) / 2 \sqrt{35}$ for which $c x(a-b)(b-c) \Phi(p)>0$ holds, but $p$ is not in $\Delta$.

By using Lemma 10, it follows from [31, Theorem 1] that the minimum of $\mu^{\prime}(\psi)$ over all unit vectors $|\psi\rangle \in \wedge^{3}(V)$ is equal to $2 / 3$, and that the minimum is attained only at the states $|\psi\rangle$ which, when regarded as a three-qubit state, are LU-equivalent to the $\mathrm{W}$ state [1]. They are all LU-equivalent to the point $p=(1 / 3,1 / 3,1 / 3,2 / 3, i \sqrt{2} / 3)$ in $\Delta$. It is easy to compute the invariants $M_{i}$ at $p$ :

$$
M_{1}=1, M_{2}=2 / 3, M_{3}=1 / 9, M_{4}=4 / 27, M_{6}=8 / 729, M_{5}=M_{7}=0 .
$$

By using Lemma 13, we deduce that the projection of $\Delta$ on the $d$-axis is the closed interval $[2 / 3,1]$. Thus if $|\psi\rangle \in \Delta$ corresponds to the point $(a, b, c, d, z)$, then $2 / 3 \leq d \leq 1$. If $d=1$ then $|\psi\rangle=e_{246}$, a decomposable 3 -vector. On the other hand, if $d=2 / 3$ then it follows easily from Eqs. (44)-(50) and (103) that $a=b=c=1 / 3$ and $z= \pm i \sqrt{2} / 3$. Thus there are exactly two points $(a, b, c, d, z) \in \Delta$ with $d=2 / 3$. Note that these points belong to $\partial \Delta$.

We shall prove now that the minimum of $\mu^{\prime}(\psi)$ over all normalized states $|\psi\rangle$ in $\wedge^{3}(V)$ is $2 / 3$. Thus, we obtain an independent proof of the fact from [31 mentioned above.

Proposition 19 Let $p=(a, b, c, d, z) \in \Delta$ where $z=x+i y$.

(i) The minimum of $d$ over all points $p \in \Delta$ is $2 / 3$. It is achieved only at the two points with coordinates $a=b=c=1 / 3, d=2 / 3$ and $z= \pm i \sqrt{2} / 3$.

(ii) $\min _{\psi} \mu(\psi)=2 / 3$, where the minimum is over all normalized states $|\psi\rangle \in \wedge^{3}(V)$.

Proof. (i) Let $p \in \Delta$ be any point where the minimum occurs. Clearly, we must have $p \in \partial \Delta$. Due to the examples given in the proposition, we have $d \leq 2 / 3$, and so $2 d^{2}-1<0$. The inequality (102) implies that $c>0$.

Assume that $x>0$. As $b x>0$, the inequalities (901)-(92) must be strict at the point $p$. Since $2 d^{2}-1<0$ and $c>0$, the inequality (93) implies that $y \neq 0$. Suppose now that the 
inequality (93) at the point $p$ is an equality, i.e., that $\Phi(p)=0$. By solving the equations $M_{1}=1$ and $\Phi(p)=0$ for $x^{2}$ and $y^{2}$, we obtain that

$$
\begin{aligned}
& 4 a b c d x^{2}=\left(d\left(d^{2}-s_{1}\right)-2 a b c\right)\left(2 a b c+d\left(2 d^{2}-1\right)\right), \\
& 4 a b c d y^{2}=\left(d\left(d^{2}-s_{1}\right)+2 a b c\right)\left(2 a b c-d\left(2 d^{2}-1\right)\right) .
\end{aligned}
$$

We choose a point $p^{\prime}=\left(a^{\prime}, b^{\prime}, c^{\prime}, d^{\prime}, z^{\prime}\right) \in \Delta, z^{\prime}=x^{\prime}+i y^{\prime}$, close to $p$ such that $a^{\prime}>a, b^{\prime}=b$, $c^{\prime}=c, d^{\prime}<d$, and $\Phi\left(p^{\prime}\right)=0$. We can do that by simply setting $d^{\prime}=d \cos \theta-a \sin \theta$ and $a^{\prime}=d \sin \theta+a \cos \theta$, where $\theta>0$ is small, and then computing $x^{\prime}$ and $y^{\prime}$ from the above two equations with $a$ and $d$ replaced by $a^{\prime}$ and $d^{\prime}$, respectively. These two equations guarantee that $\Phi\left(p^{\prime}\right)=0$. As $x>0$ and $y \neq 0$, we have to choose $x^{\prime}>0$, and $y^{\prime}$ to have the same sign as $y$. For sufficiently small $\theta>0$, the inequalities (90)-(92) will be satisfied at the point $p^{\prime}$, and we will have equality in (93). As $b^{\prime}=b$ and $c^{\prime}=c$, the other inequalities defining $\Delta$ will also be satisfied. This contradicts with the hypothesis that $d$ takes the minimal value at the point $p$. We conclude that also the inequality (93) is strict at $p$.

Consider the function $f(t)=1-s_{1}-y^{2}-(x+t)^{2}$ of a real variable $t$. At the point $t=0$ we have $f(0)=d^{2}$. Note that $x<1$ and $f^{\prime}(0)=-2 x<0$. Hence we can choose a small $\varepsilon>0$ such that $f(\varepsilon)<d^{2}$ and all four inequalities (90)-(93) are still valid at the point $q=(a, b, c, \sqrt{f(\varepsilon)}, x+\varepsilon+i y)$. By part (b) of Theorem 16, we conclude that $q \in \Delta$. This contradicts the fact that the minimum of the coordinate $d$ over $\Delta$ occurs at the point $p$. Thus we have shown that $x=0$.

By the inequalities (91) and (102), we have $d\left(d^{2}-s_{1}\right) \geq 2 a b c \geq d\left(1-2 d^{2}\right)$, and so $c>0$ and $s_{1} \leq 3 d^{2}-1$. By the arithmetic-geometric mean inequality we have

$$
\left(\frac{3 d^{2}-1}{3}\right)^{3} \geq\left(\frac{s_{1}}{3}\right)^{3} \geq(a b c)^{2} \geq \frac{d^{2}}{4}\left(1-2 d^{2}\right)^{2} .
$$

By expanding the leftmost and rightmost member, we obtain that $d \geq 2 / 3$. Moreover, if $d=2 / 3$ then the above inequalities become equalities, and so we must have $a=b=c$. By (i) we have $a \geq 1 / 3$ and from (91) we have $a \leq 1 / 3$. Hence, $p=(1,1,1,2, \pm i \sqrt{2}) / 3$.

(ii) Since $\mu^{\prime}$ is LU-invariant, by Lemma 13 we may minimize over $\Delta$ only. By Lemma 10 we have $\mu^{\prime}=\mu$ on $\Delta$. Hence (ii) follows from (i).

\section{Three-fermion canonical form}

We begin by introducing the notation $p \mapsto p^{\prime}$ for the projection map $\mathbb{R}^{4} \times \mathbb{C} \rightarrow \mathbb{R}^{4}$. Thus for any point $p=(a, b, c, d, z) \in \mathbb{R}^{4} \times \mathbb{C}$, we set $p^{\prime}=(a, b, c, d)$. Let $\Delta^{\prime}$ be the image of $\Delta$ under this projection map, i.e., $\Delta^{\prime}=\left\{p^{\prime}: p \in \Delta\right\}$.

\subsection{The canonical form}

In order to state the main result of this section, the canonical form for fermionic states, we have to resolve the question of LU-equivalence of points in $\Delta$. The most important fact is that two distinct points in $\Delta$ which are LU-equivalent must lie on $\partial \Delta$. 
Proposition 20 Let $p=(a, b, c, d, z)$ and $q=(\tilde{a}, \tilde{b}, \tilde{c}, \tilde{d}, \tilde{z})$ be distinct points of $\Delta$, and let $z=x+i y$ and $\tilde{z}=\tilde{x}+i \tilde{y}$. Then $p$ and $q$ are $L U$-equivalent if and only if the following two conditions hold

(i) $p^{\prime}=q^{\prime}$ and also $\tilde{x}=x$ if $c>0$;

(ii) $c x \Phi(p)=\tilde{c} \tilde{x} \Phi(q)=0$.

In particular, if $p$ and $q$ are $L U$-equivalent, then $p, q \in \partial \Delta$.

Proof. Necessity. Since $p$ and $q$ are LU-equivalent, they have the same values of the invariants $M_{i}, i=1, \ldots, 7$. It follows from Definition 11 (ii) that $\tilde{d}=d$. By substituting $|z|^{2}$ with $t$ on the left hand side of Eq. (118), we obtain a cubic polynomial $g(t)$. As $|z|^{2}$ is one of its roots, $g(t)$ factorizes as $g(t)=3 d^{2}\left(t-|z|^{2}\right) h(t)$ where

$$
h(t)=d^{2} t^{2}-d^{2}\left(3-6 d^{2}-|z|^{2}\right) t+d^{2}\left(2 d^{2}-1\right)\left(4 d^{2}-2+|z|^{2}\right)-8 s_{3} .
$$

As the discriminant of $h(t)$ is nonnegative, $h(t)$ has two real roots $t_{1} \leq t_{2}$. As $t_{1}+t_{2}=$ $3-6 d^{2}-|z|^{2}$, we have $2|z|^{2}-\left(t_{1}+t_{2}\right)=6 d^{2}+3|z|^{2}-3=3\left(d^{2}-s_{1}\right) \geq 0$ by Theorem 16. Hence, $|z|^{2} \geq\left(t_{1}+t_{2}\right) / 2$. On the other hand, $h\left(|z|^{2}\right)=2\left(d^{2}\left(d^{2}-s_{1}\right)^{2}-4 s_{3}\right)$ is nonnegative by the same theorem. It follows that $|z|^{2} \geq t_{2}$. Thus all roots of $g(t)$ are real, and $|z|^{2}$ is the largest root. By the same argument, $|\tilde{z}|^{2}$ is the largest root of $g(t)$, and so $|\tilde{z}|=|z|$.

Eqs. (115) -(117) imply that the elementary symmetric functions $s_{1}, s_{2}, s_{3}$ of $a, b, c$ are the same as those of $\tilde{a}, \tilde{b}, \tilde{c}$. As $a \geq b \geq c \geq 0$ and $\tilde{a} \geq \tilde{b} \geq \tilde{c} \geq 0$, it follows that $\tilde{a}=a, \tilde{b}=b$ and $\tilde{c}=c$. Consequently, we have $p^{\prime}=q^{\prime}$. If $c>0$, then the inequalities $x \geq 0, \tilde{x} \geq 0$, and Eq. (46) imply that $\tilde{x}=x$. Thus (i) holds.

Assume that $c x \neq 0$. By (i) we have $\tilde{x}=x$ and $\tilde{y}=-y \neq 0$. Since $M_{7}$ has the same value at $p$ and $q$ and $\tilde{a} \tilde{b} \tilde{c} \tilde{d} \tilde{x} \tilde{y}=-a b c d x y \neq 0$, Eq. (50) implies that $\Phi(q)=-\Phi(p)$. By Theorem 16 both $\Phi(p)$ and $\Phi(q)$ are nonnegative, and so $\Phi(q)=\Phi(p)=0$. Hence, (ii) holds.

Sufficiency. We have to show that $M_{i}(p)=M_{i}(q)$ for $i=1, \ldots, 7$. This follows immediately by inspection of the formulae (44)-(50).

Finally, note that if $p$ and $q$ are LU-equivalent, then (ii) and Lemma 18 imply that $p, q \in \partial \Delta$.

Let us denote by $\mathcal{O}_{\psi}$ the LU-orbit of $|\psi\rangle \in \wedge^{3}(V)$. Thus, $\mathcal{O}_{\psi}=\mathrm{U}(6) \cdot|\psi\rangle=\{g \cdot|\psi\rangle: g \in$ $\mathrm{U}(6)\}$. Assume that $\|\psi\|=1$. The intersection $\mathcal{O}_{\psi} \cap \Delta$ consists of a single point if and only if one of the following holds:

(i) $c x \Phi(p)>0$;

(ii) $c>0$ and $x \Phi(p)=y=0$.

(iii) $c=z=0$.

Otherwise, $\mathcal{O}_{\psi} \cap \Delta$ is either

(iv) a pair of points: $\{p, q\}$ where $p=(a, b, c, d, z), q=\left(a, b, c, d, z^{*}\right), c y>0$, and $x \Phi(p)=0$; or 
(v) a semicircle: $\left\{\left(a, b, 0, d, r e^{i t}\right):|t| \leq \pi / 2\right\}$ where $r=\sqrt{1-a^{2}-b^{2}-d^{2}}>0$.

While the case (i) covers all points in $\Delta^{0}$ and some points on $\partial \Delta$, in all other cases the points lie on $\partial \Delta$. It is easy to see that all five cases indeed occur.

We now state our main result, which follows immediately from Lemma 13 and Proposition 20.

Theorem 21 Any normalized pure fermionic state $|\varphi\rangle \in \wedge^{3}(V)$, with $\operatorname{dim} V=6$, is $L U$ equivalent to a state

$$
|\psi\rangle=a e_{235}+b e_{145}+c e_{136}+d e_{246}+z e_{135}
$$

where $p:=(a, b, c, d, z) \in \Delta, z=x+i y$. Such a state $|\psi\rangle$ is unique if $\operatorname{cx} \Phi(p)>0$; in particular this is true if $p \in \Delta^{0}$. To guarantee the uniqueness of $|\psi\rangle$ when $\operatorname{cx} \Phi(p)=0$, we require that (i) $y \geq 0$ and (ii) $y=0$ if $c=0$.

(For a simpler description of the canonical region $\Delta$ see Proposition 23 below.)

Our next objective is to give a more geometric description of the canonical region $\Delta$ and its projection $\Delta^{\prime}$. By restricting the projection map $\mathbb{R}^{4} \times \mathbb{C} \rightarrow \mathbb{R}^{4}$, we obtain the map $\Delta \rightarrow \Delta^{\prime}, p \mapsto p^{\prime}$. The fibre of this map over a point $p^{\prime}:=(a, b, c, d) \in \Delta^{\prime}$ is the set $F_{p^{\prime}}:=\left\{q \in \Delta: q^{\prime}=p^{\prime}\right\}$. We shall determine the nature of these fibres and find explicit inequalities which define $\Delta^{\prime}$ as a subset of $\mathbb{R}^{4}$.

For convenience, we set $r:=\sqrt{1-a^{2}-b^{2}-c^{2}-d^{2}}$ and denote by $S_{p^{\prime}}$ the semicircle consisting of all points $\left(p^{\prime}, z\right)=(a, b, c, d, z)$ with $z=x+i y$ such that $|z|=r$ and $x \geq 0$. Note that $F_{p^{\prime}}=S_{p^{\prime}} \cap \Delta \supseteq\{p\}$ for all $p \in \Delta$. If $c=0$ it is easy to see that $F_{p^{\prime}}=S_{p^{\prime}}$. Note that $S_{p^{\prime}}=\{p\}$ if and only if $r=0$.

We claim that if $F_{p^{\prime}}=\{p\}$, then $r=0$. Indeed, observe that $p=\left(p^{\prime}, z\right) \in \Delta$, with $z=x+i y$, implies that $\left(p^{\prime}, z^{*}\right) \in \Delta$, and so we must have $y=0$. Let $q:=\left(p^{\prime}, i r\right) \in S_{p^{\prime}}$. Since $p \in \Delta$, the inequalities (90)-(93) are valid at $p$. The first two do not involve $z$ and remain valid at $q$. By using the fact that $y=0$, we see that the remaining two inequalities remain valid when we replace $p$ by $q$. Hence, Theorem [16 implies that $q \in \Delta$. Consequently, $q \in F_{p^{\prime}}$, and so we must have $q=p$, i.e., $r=0$.

Finally, let us denote by $\Psi(a, b, c, d, z)$ the left hand side of (92). It is evident from the definitions of $\Phi$ and $\Psi$ that $\Phi\left(a, b, c, d, r e^{i t}\right)$ and $\Psi\left(a, b, c, d, r e^{i t}\right)$, considered as functions of $t \in[0, \pi / 2]$, are constant if $c=0$, and are strictly increasing if $c>0$.

Lemma 22 Let $p=\left(a, b, c, d, r e^{i \theta}\right) \in \Delta, r>0,|\theta|<\pi / 2$. The following assertions hold:

(i) If $\Phi\left(p^{\prime}, r\right) \geq 0$ then $F_{p^{\prime}}=S_{p^{\prime}}$.

(ii) If $\Phi\left(p^{\prime}, r\right)<0$ then $F_{p^{\prime}}$ consists of all points $\left(p^{\prime}, z^{\prime}\right) \in S_{p^{\prime}}$, $z^{\prime}=x^{\prime}+i y^{\prime}$, such that $0 \leq x^{\prime} \leq x_{0}$ where $x_{0}$ is the nonnegative solution of Eq. (104).

Proof. Without any loss of generality, we may assume that $\theta \geq 0$. For $t \in[0, \pi / 2]$, let $p(t)=\left(p^{\prime}, r e^{i t}\right)$. If $c=0$, then the functions $\Phi(p(t))$ and $\Psi(p(t))$ are constant and so (i) 
holds in that case. We assume that $c>0$. The inequalities (90) and (91) are valid at all points of the fibre $F_{p^{\prime}}$, not only at the point $p$, because their left hand sides do not depend on $z$. Moreover, since $b>0$ these two inequalities are strict at each point $p(t) \in F_{p^{\prime}}$ with $0 \leq t<\pi / 2$.

(i) We have to prove that $p(t) \in \Delta$ for $t \in[0, \pi / 2]$. The proof is based on part (a) of Theorem 16. As $p \in \Delta$, we have $\Psi(p(\theta))=\Psi(p) \geq 0$. Assume that $\Psi(p(0))<0$ and so $p(0) \notin \Delta$. There is a unique $t_{1} \in(0, \theta]$ such that $\Psi\left(p\left(t_{1}\right)\right)=0$. As $\Phi\left(p\left(t_{1}\right)\right)>0$, we have $p\left(t_{1}\right) \in \Delta$. Since $b>0$ and $0<t_{1}<\pi / 2$, we must have $\Psi\left(p\left(t_{1}\right)\right)>0$ by part (a) of Theorem [16. Hence, we have a contradiction. We conclude that $\Psi(p(0)) \geq 0$. It follows that for any $t \in[0, \pi / 2]$, we have $\Phi(p(t)) \geq 0$ and $\Psi(p(t)) \geq 0$, and so $p(t) \in \Delta$.

(ii) Since $p \in \Delta$, we have $\Phi(p) \geq 0$ and $\Psi(p) \geq 0$. On the other hand, by the hypothesis, we have $\Phi\left(p^{\prime}, r\right)<0$. It follows that there is a unique $t_{1} \in(0, \theta]$ such that $\Phi\left(p\left(t_{1}\right)\right)=0$. We deduce that $x_{0}=r \cos t_{1}$, and that $\Phi(p(t)) \geq 0$ if and only if $t_{1} \leq t \leq \pi / 2$. If $\Psi\left(p\left(t_{1}\right)\right)<0$, we can deduce a contradiction by the same argument as in part (i). Therefore $\Psi\left(p\left(t_{1}\right)\right) \geq 0$, and the assertion (ii) follows easily.

We now determine the projection $\Delta^{\prime}$ of $\Delta$ and simplify the set of inequalities in Theorem 16. which define $\Delta$.

\section{Proposition 23}

(i) The subset $\Delta^{\prime} \subseteq \mathbb{R}^{4}$ consists of all points $p^{\prime}=(a, b, c, d)$ which satisfy the inequalities $a \geq b \geq c \geq 0, d>0, a^{2}+b^{2}+c^{2}+d^{2} \leq 1$ as well as the inequalities (91) and (102).

(ii) $A$ point $p=(a, b, c, d, z) \in \mathbb{R}^{4} \times \mathbb{C}$ belongs to $\Delta$ if and only if $a^{2}+b^{2}+c^{2}+d^{2}+|z|^{2}=1$ and $p$ satisfies the following inequalities (with $s_{1}=a^{2}+b^{2}+c^{2}$ )

$$
\begin{aligned}
& a \geq b \geq c \geq 0, x \geq 0, d>0 \\
& d\left(d^{2}-s_{1}\right)-2 a b c \geq 0 \\
& 2 a b c-d\left(1-2 d^{2}\right) \geq 0 \\
& 4 a b c d x^{2} \leq\left(d\left(d^{2}-s_{1}\right)-2 a b c\right)\left(2 a b c-d\left(1-2 d^{2}\right)\right) .
\end{aligned}
$$

Proof. (i) Necessity. Let $p^{\prime}:=(a, b, c, d) \in \Delta^{\prime}$ and let $r=\sqrt{1-s_{1}-d^{2}}$. It follows from Lemma 22 that the point $p:=\left(p^{\prime}, i r\right) \in F_{p^{\prime}}$. Consequently, $p^{\prime}$ must satisfy all inequalities listed in the proposition.

Sufficiency. Let $p=(a, b, c, d, i r)$, where $r=\sqrt{1-s_{1}-d^{2}} \geq 0$. It suffices to show that $p \in \Delta$. By Theorem [16, it suffices to verify that $p$ satisfies the inequalities (90), (92) and (93). As $d>0$, the inequality (90) follows immediately from (111). One can easily verify that

$$
\Psi(p)-\Phi(p)=(a b-c d)^{2}\left(3 d^{2}-1-s_{1}\right)+\left(d\left(d^{2}-s_{1}\right)+2 a b c\right)\left(d\left(2 d^{2}-1\right)+2 a b c\right) .
$$

The inequalities (110) and (111) imply that $3 d^{2}-1-s_{1} \geq 0$, and so $\Psi(p)-\Phi(p) \geq 0$. Since at the point $p$ we have $z=i r$ and so $x=0$, we obtain that $\Phi(p)=\left(d\left(d^{2}-s_{1}\right)-\right.$ 
$2 a b c)\left(2 a b c+d\left(2 d^{2}-1\right)\right)$. Thus, the inequalities (110) and (111) also imply that $\Phi(p) \geq 0$. Thus $\Psi(p) \geq \Phi(p) \geq 0$, and so the inequalities (92) and (93) also hold at the point $p$.

(ii) We already know that the listed inequalities are necessary. (Note that the inequalities (93) and (112) are equivalent to each other.) To prove the sufficiency, we observe that all inequalities listed in part (i) are satisfied at the point $p^{\prime}:=(a, b, c, d)$, and so $p^{\prime} \in \Delta^{\prime}$. Since $|z|=r:=\sqrt{1-s_{1}-d^{2}}$ and $x \geq 0$, we have $z \in S_{p^{\prime}}$, i.e., $z=r e^{i t}$ for some real $t,|t| \leq \pi / 2$. As $p$ satisfies the inequality (112), we have $\Phi\left(p^{\prime}, r e^{i t}\right)=\Phi(p) \geq 0$. Now Lemma 22 implies that $p \in F_{p^{\prime}}$ and, in particular, $p \in \Delta$.

We point out that none of the four inequalities $d>0$, and (110)-(112) in part (ii) can be omitted. For the inequality $d>0$ we can use the point $p=(3,0,0,0,4) / 5$ which is not in $\Delta$ and satisfies the other three inequalities. Similarly, for the remaining three inequalities: $(1,1,1,1,0) / 2$ violates only (110), $(1,0,0,1,1) / \sqrt{3}$ violates only (111), and $(4,2,2,6,2+3 i) / \sqrt{73}$ violates only (112) .

Note also that in view of Proposition 19, the inequality $d>0$ can be replaced by the stronger inequality $d \geq 2 / 3$.

\subsection{The algorithm}

We shall describe a numerical algorithm which, for a generic normalized state $|\phi\rangle \in \wedge^{3}(V)$ as input, computes its canonical form $|\psi\rangle$. Let us denote by $p=(a, b, c, d, z)$ the point in $\Delta$ that corresponds to $|\psi\rangle$. So, our problem is to compute the coordinates $a, b, c, d$ and $z=x+i y$. Since $|\phi\rangle$ is given, we can compute the values of the invariants $M_{i}$ at $|\phi\rangle$. For simplicity, we shall write in this subsection $M_{i}=M_{i}(\phi)$. Since $|\psi\rangle$ is LU-equivalent to $|\phi\rangle$, we also have $M_{i}(p)=M_{i}$ for $i=1, \ldots, 7$. Thus, $p=(a, b, c, d, z)$ is a solution of this system of equations. In order to solve numerically this system of equations, we proceed as follows.

By eliminating the expression $x^{2}-y^{2}$ from Eqs. (46) and (48) we obtain

$$
3 M_{5}+4 d^{2} M_{3}=3 d^{4}|z|^{4}+24\left(s_{3}-s_{2} d^{2}\right) d^{2}+4\left(s_{2}+s_{1} d^{2}\right) d^{2} .
$$

By solving Eqs. (44), (45) and (114) for $s_{1}, s_{2}$, and $s_{3}$, we obtain that

$$
\begin{aligned}
s_{1}= & 1-d^{2}-|z|^{2} \\
2 s_{2}= & 2 d^{4}-\left(2+|z|^{2}\right) d^{2}+M_{2}, \\
24 d^{2} s_{3}= & 24 d^{8}-12\left(2+|z|^{2}\right) d^{6}+3\left(4 M_{2}+2|z|^{2}-|z|^{4}\right) d^{4} \\
& \quad+2\left(2 M_{3}-M_{2}\right) d^{2}+3 M_{5} .
\end{aligned}
$$

As $d>0$, by substituting the above expressions into Eqs. (47) and (49) we obtain the 
equations

$$
\begin{aligned}
& 3 d^{4}|z|^{6}+9\left(2 d^{2}-1\right) d^{4}|z|^{4}+\left(6\left(1-2 M_{2}\right) d^{4}+2\left(M_{2}-2 M_{3}\right) d^{2}-3 M_{5}\right)|z|^{2} \\
& \quad-96 d^{10}+144 d^{8}-48\left(1+M_{2}\right) d^{6}+16\left(2 M_{2}-M_{3}\right) d^{4} \\
& \quad+2\left(2 M_{3}+3 M_{2}^{2}-M_{2}-12 M_{4}-6 M_{5}\right) d^{2}+3 M_{5}=0 \\
& 27 d^{8}|z|^{8}+36 d^{6}\left(8 d^{4}-4 d^{2}+M_{2}\right)|z|^{6} \\
& \quad+6 d^{4}\left(144 d^{8}-144 d^{6}+6 d^{4}\left(7+4 M_{2}\right)-4 d^{2}\left(4 M_{2}+M_{3}\right)-3 M_{5}\right)|z|^{4} \\
& \quad+12 d^{2}\left(4 d^{4}-2 d^{2}+M_{2}\right)\left(6 d^{4}\left(1-2 M_{2}\right)+2 d^{2}\left(M_{2}-2 M_{3}\right)-3 M_{5}\right)|z|^{2} \\
& \quad-2304 d^{16}+4608 d^{14}-576\left(5+4 M_{2}\right) d^{12}+192\left(3+16 M_{2}-2 M_{3}\right) d^{10} \\
& \quad+96\left(4 M_{3}-11 M_{2}-6 M_{2}^{2}-3 M_{5}\right) d^{8}+48\left(M_{2}-2 M_{3}+8 M_{2}^{2}+6 M_{5}-4 M_{2} M_{3}\right) d^{6} \\
& \quad+4\left(16 M_{2} M_{3}-7 M_{2}^{2}-18 M_{5}-36 M_{2} M_{5}-4 M_{3}^{2}-144 M_{6}\right) d^{4} \\
& \quad+24\left(2 M_{2}-M_{3}\right) M_{5} d^{2}-9 M_{5}^{2}=0 .
\end{aligned}
$$

By substituting formally $|z|^{2}$ and $d^{2}$ in these two equations with new indeterminates $s$ and $t$, respectively, we obtain two polynomials $g(s, t)$ and $h(s, t)$ which have a common solution, namely $s=|z|^{2}, t=d^{2}$. We can eliminate now $s$ from these two equations by computing the resultant of the polynomials $g(s, t)$ and $h(s, t)$ with respect to the variable $s$. This resultant has the form $-2^{4} 3^{5} t^{12} f(t)$, where $f(t)$ is a polynomial in $t$ of degree 8 whose coefficients are polynomials in the six parameters $M_{1}, \ldots, M_{6}$ with integer coefficients. As the fully expanded $f(t)$ has 550 terms, it is only given in Appendix A. Since we know that $t=d^{2}$ is a root of $f(t)$, the polynomial $f(t)$ has a factorization $f(t)=\left(t-d^{2}\right) f_{1}(t)$. It turns out that $f_{1}(t)$, when expanded as a polynomial in $t$ and the parameters $M_{i}$, has 41967 terms.

After substituting the expressions (44)-(49) into $f_{1}(t)$, by using Maple we obtain the following factorization

$$
f_{1}\left(d^{2}\right)=2^{8} 3^{5}(a b c d)^{2}\left(d^{2}-a^{2}\right)\left(d^{2}-b^{2}\right)\left(d^{2}-c^{2}\right)\left(d^{2}\left(d^{2}-s_{1}\right)^{2}-4 s_{3}\right)^{3} \cdot \Phi(p) .
$$

Hence, $d^{2}$ is a multiple root of $f(t)$ if and only if $c=0$ or $d\left(d^{2}-s_{1}\right)-2 a b c=0$ or $\Phi(p)=0$. Recall that for $p \in \Delta$ the equality $d\left(d^{2}-s_{1}\right)-2 a b c=0$ implies that $b x=0$. It follows that $d^{2}$ is a multiple root of $f(t)$ if and only if $c x \Phi(p)=0$.

Computing $d^{2}$ is the main step in the algorithm. We applied our algorithm to about 100 randomly chosen input states $|\phi\rangle$. In all these cases the equation $f(t)=0$ had 4 , 6 , or 8 real roots, all of them lying in the interval $(0,1)$. Moreover, $d^{2}$ was always the largest of these real roots. Since $p \in \Delta$, the correct value of $d$ will be equal to $\mu(\phi)$.

Once the value of $d^{2}$ is selected, the remaining steps of the algorithm are straightforward. We compute $|z|^{2}$ by solving the polynomial equation $g\left(s, d^{2}\right)=0$. As shown in the proof of Proposition 20, this cubic has three real roots and $|z|^{2}$ is its largest root. Then the equations (115) -(117) provide the values of $s_{1}, s_{2}$, and $s_{3}$. As $a^{2}, b^{2}, c^{2}$ are the roots of the cubic $t^{3}-s_{1} t^{2}+s_{2} t-s_{3}$, and we know that $a \geq b \geq c \geq 0$, we can compute $a, b$, and $c$. Next, $x^{2}$ (and $y^{2}$ ) can be computed from Eq. (46). As $x \geq 0$, we obtain also the value of $x$. Finally, we can determine the sign of $y$ by using Eq. (50). 
When applying the algorithm, we had to increase the precision by setting the global Maple parameter Digits from 10 (the default) to 200 since some of the coefficients in $f(t)=0$ are small and hence the equation might not be well conditioned.

We conjecture that, if $f(t)$ is not identically zero, then

(1) all real roots of $f(t)$ belong to the interval $[0,1]$ and

(2) $d^{2}$ is the largest real root of $f(t)$.

\section{Real and quasi-real pure fermionic states}

We say that a state $|\psi\rangle \in \wedge^{3}(V)$ is real if there exists $g \in \mathrm{U}(6)$ such that all components of $g \cdot|\psi\rangle$, with respect to the standard basis $\left\{e_{i j k}\right\}$, are real. Let us denote by $\mathcal{O}_{\psi}$ the LU-orbit containing the state $|\psi\rangle$, i.e., $\mathcal{O}_{\psi}=\mathrm{U}(6) \cdot|\psi\rangle=\{g \cdot|\psi\rangle: g \in \mathrm{U}(6)\}$. By $\left|\psi^{*}\right\rangle$ we denote the complex conjugate of $|\psi\rangle$ computed in the standard basis. We say that $|\psi\rangle$ is quasi-real if $\left|\psi^{*}\right\rangle$ and $|\psi\rangle$ are LU-equivalent. It is immediate that $|\psi\rangle$ is quasi-real if and only if $\mathcal{O}_{\psi^{*}}=\mathcal{O}_{\psi}$. Note that every real state is quasi-real.

One defines similarly the real and quasi-real pure states of three qubits. A pure threequbit state is real if in some orthonormal basis all of its components are real numbers. It is quasi-real if it is LU-equivalent to its complex conjugate state. It was proved in [11, Eq. (34) and Appendix B] that the pure three-qubit quasi-real states are real.

By using the invariants, we can characterize the quasi-real states.

Proposition 24 A state $|\psi\rangle \in \wedge^{3}(V)$ is quasi-real if and only if $M_{7}(\psi)=0$.

Proof. Clearly we may assume that $\|\psi\|=1$. Since $M_{7}$ is a unitary invariant, we may replace $|\psi\rangle$ with any state in the orbit $\mathcal{O}_{\psi}$. Hence, by Lemma 13 we may assume that $|\psi\rangle \in \Delta$ and that it is given by Eq. (18). Thus $|\psi\rangle$ corresponds to the point $(a, b, c, d, z) \in \Delta$ and $\left|\psi^{*}\right\rangle$ corresponds to the point $\left(a, b, c, d, z^{*}\right) \in \Delta$. It is obvious from Eqs. (44)-(50) that $M_{i}(\psi)=M_{i}\left(\psi^{*}\right)$ for $i=1, \ldots, 6$ and $M_{7}\left(\psi^{*}\right)=-M_{7}(\psi)$. Now the assertion follows from Corollary 7 .

We shall need the following simple lemma.

Lemma 25 Let $p=(a, b, c, d, z) \in \Delta, c>0$, and let $D_{a}, D_{b}, D_{c}$ be defined as in Sec. 3 . Then we have $D_{c} \geq D_{b} \geq D_{a}>0$. Moreover, $D_{a}=D_{b}$ if and only if $a=b$, and $D_{b}=D_{c}$ if and only if $b=c$. Consequently, the determinants $D_{a}, D_{b}, D_{c}$ are uniquely determined by the invariants $M_{2}, M_{4}, M_{6}$.

Proof. As $c>0$, we have $d>a \geq b \geq c>0$. The assertions of the lemma follow from the formulae $D_{c}-D_{b}=\left(d^{2}-a^{2}\right)\left(b^{2}-c^{2}\right)$ and $D_{b}-D_{a}=\left(d^{2}-c^{2}\right)\left(a^{2}-b^{2}\right)$, together with Eqs. (33)-(35).

We can now extend the above mentioned result of Acin et al. [11] to the fermionic states in $\wedge^{3}(V)$.

Proposition 26 Any quasi-real state $|\psi\rangle \in \wedge^{3}(V)$ is real. 
Proof. By Lemma 13, we may assume that $|\psi\rangle \in \Delta$. Let $p=(a, b, c, d, z)$ be the point corresponding to $|\psi\rangle$. If $c=0$, then we may assume that $z=x \geq 0$ and so $|\psi\rangle$ is real. Thus we may assume that $c>0$.

As $|\psi\rangle$ is quasi-real, we have $\left|\psi^{*}\right\rangle \in \mathcal{O}_{\psi}$. By Theorem 6, the intersection $W \cap \mathcal{O}_{\psi}$ is a single $G$-orbit. Since both $|\psi\rangle$ and $\left|\psi^{*}\right\rangle$ belong to this intersection, there exists $g \in G$ such that $\left|\psi^{*}\right\rangle=g \cdot|\psi\rangle$. By using that $g=U \sigma$ for some $U \in \mathrm{U}(2) \times \mathrm{U}(2) \times \mathrm{U}(2)$ and some $\sigma \in S_{3}$, we obtain that $\sigma^{-1} U \sigma \cdot|\psi\rangle=\sigma^{-1} \cdot\left|\psi^{*}\right\rangle$. Note that $\sigma^{-1} U \sigma \in \mathrm{U}(2) \times \mathrm{U}(2) \times \mathrm{U}(2)$. Since the invariants $M_{2}, M_{4}, M_{6}$ take the same values at $|\psi\rangle$ and $\left|\psi^{*}\right\rangle$, Lemma 25 implies that $\sigma^{-1} \cdot\left|\psi^{*}\right\rangle=\left|\psi^{*}\right\rangle$. We can now apply the above mentioned result of Ref. [1] to conclude the proof.

\section{A new canonical form for pure three-qubit states}

A canonical form for pure three-qubit states was constructed first by Acin et al. in [11]. Their Fig. 1 shows three inequivalent set of states (I), (II), and (III) that can be used to construct a canonical form. Their canonical form is constructed by using the set (III). They also show that case (II) can be reduced to case (III). However, they were not able to handle the symmetric decomposition (I). By using our canonical form for three fermions, we can obtain a solution in that case.

Recall that $\Sigma$ denotes the unit sphere of $W_{6}$ defined by the equation $a^{2}+b^{2}+c^{2}+d^{2}+|z|^{2}=$ 1. We begin by introducing the region

$$
\Theta=\bigcup_{\sigma \in S_{3}} \sigma \cdot \Delta \subseteq \Sigma
$$

which is obviously invariant under the action of $S_{3}$ (for the latter see Sec. 31).

Since the inequalities (110), (111), and (112) are not affected by the permutations of the variables $a, b, c$, it follows easily that $\Theta$ is the region of $\Sigma$ defined by these three inequalities and the linear inequalities $a, b, c, x \geq 0$ and $d>0$. One can also show that the relative interior $\Theta^{0}$ of $\Theta$ consists of the points of $\Theta$ at which all the inequalities defining $\Theta$ are strict. It follows from Theorem [16 that if at some point $p=(a, b, c, d, z) \in \Theta$ the equality holds in (110) or (111), then we must have $a b c x=0$. Consequently, the relative boundary $\partial \Theta$ of $\Theta$ consists of the points $p=(a, b, c, d, z) \in \Theta$ for which $a b c x \Phi(p)=0$.

Let us give yet another description of the region $\Theta$. Recall that $\Sigma_{i}$ denotes the unit sphere in the subspace $V_{i} \subseteq V$.

Proposition 27 The set $\Theta$ consists of all points $p=(a, b, c, d, z) \in \Sigma, z=x+i y$, satisfying the inequalities $a, b, c, x \geq 0$ and such that

$$
d=\max _{\alpha, \beta, \gamma}|\langle\alpha, \beta, \gamma \mid \psi\rangle|, \quad(\alpha, \beta, \gamma) \in \Sigma_{1} \times \Sigma_{2} \times \Sigma_{3} .
$$

Proof. If $p=(a, b, c, d, z) \in \Theta$, then $p \in \sigma \cdot \Delta$ for some $\sigma \in S_{3}$. As $S_{3}$ only permutes the coordinates $a, b, c$ and leaves the coordinates $d$ and $z$ unchanged, we have $a, b, c, x \geq 0$. Now (122) follows form Lemma 10 and Definition 11 . 
Evidently, two LU-equivalent three-qubit states in $\Theta$ have the same coefficient $d$.

To prove the next proposition, we shall use the invariants $Q_{i}, 1 \leq i \leq 7$, of three qubits which are described in Sec. 5. At a point $p=(a, b, c, d, z) \in W_{6}$, we have

$$
\begin{aligned}
& Q_{2}-Q_{3}=2\left(a^{2}-b^{2}\right)\left(d^{2}-c^{2}\right), \\
& Q_{3}-Q_{4}=2\left(b^{2}-c^{2}\right)\left(d^{2}-a^{2}\right), \\
& Q_{4}-Q_{2}=2\left(c^{2}-a^{2}\right)\left(d^{2}-b^{2}\right) .
\end{aligned}
$$

The following proposition gives the canonical form for pure three-qubit states that was mentioned in [1] but left open.

Proposition 28 Any normalized pure three-qubit state $|\phi\rangle$ is LU-equivalent to a state

$$
|\psi\rangle=a|100\rangle+b|010\rangle+c|001\rangle+d|111\rangle+z|000\rangle,
$$

where $p:=(a, b, c, d, z) \in \Theta, z=x+i y$. Such state is unique if abcx $\Phi(p)>0$, i.e., if $p \in \Theta^{0}$. To guarantee the uniqueness of $|\psi\rangle$ when abcx $\Phi(p)=0$, i.e., when $p \in \partial \Theta$, we require that (i) $y \geq 0$ and (ii) $y=0$ if $a b c=0$.

Proof. First we show that $|\phi\rangle$ is LU-equivalent to some state in $\Theta$. By Lemma 13 we can transform $|\phi\rangle$ into $\Delta$ by the group $G$, i.e., there exist $\sigma \in S_{3}$ and $g \in \mathrm{U}(2) \times \mathrm{U}(2) \times \mathrm{U}(2)$ such that $|\chi\rangle:=\sigma g \cdot|\phi\rangle \in \Delta$. Consequently, $|\psi\rangle:=g \cdot|\phi\rangle \in \sigma^{-1} \cdot \Delta \subseteq \Theta$.

In order to prove the uniqueness assertions, suppose that we have two distinct points $p=(a, b, c, d, z)$ and $q=(\tilde{a}, \tilde{b}, \tilde{c}, \tilde{d}, \tilde{z})$ in $\Theta$, which are equivalent under the local unitary group $\mathrm{U}(2) \times \mathrm{U}(2) \times \mathrm{U}(2)$ of three qubits. By definition of $\Theta$, there exist $\sigma, \sigma^{\prime} \in S_{3}$ such that $\sigma \cdot p, \tilde{\sigma} \cdot q \in \Delta$. Note that the points $\sigma \cdot p$ and $\tilde{\sigma} \cdot q$ belong to the same orbit of $G$. Since $S_{3}$ only permutes the coordinates $a, b, c$ and leaves the coordinates $d$ and $z$ unchanged, Proposition 20 implies that $\tilde{d}=d$ and that $(\tilde{a}, \tilde{b}, \tilde{c})$ is a permutation of $(a, b, c)$. Since $Q_{i}(p)=Q_{i}(q)$ for all $i$, it follows from Eqs. (123)-(125) that $\tilde{a}=a, \tilde{b}=b, \tilde{c}=c$ and consequently $|\tilde{z}|=|z|$. As usual, we write $z=x+i y$ and $\tilde{z}=\tilde{x}+i \tilde{y}$. If $a b c>0$, then Proposition 20 also implies that $\tilde{x}=x$, and so $\tilde{y}=-y \neq 0$ because $p \neq q$. If $a b c=0$, then the invariants $Q_{i}(p)$ depend only on $a, b, c, d$ and $|z|^{2}$, and so all points $\left(a, b, c, d, r e^{i t}\right)$, where $r=\sqrt{1-s_{1}-d^{2}}$ and $|t| \leq \pi / 2$, are LU-equivalent to each other. All uniqueness assertions easily follow.

\section{Acknowledgments}

We thank Jianxin Chen, Zhengfeng Ji, and Mary Beth Ruskai for helpful discussion on this paper. LC was mainly supported by MITACS and NSERC. The CQT is funded by the Singapore MoE and the NRF as part of the Research Centres of Excellence programme. DD was supported in part by an NSERC Discovery Grant. BZ is supported by NSERC and CIFAR. The computations involving invariants were performed by using Maple ${ }^{\mathrm{TM}}$ [41] and Magma 42. 


\section{A The polynomial $f(t)$}

The polynomial $f(t)$ defined in Sec. 7.2 can be written as

$$
f(t)=\sum_{k=0}^{8} c_{k} t^{k}
$$

where

$$
\begin{aligned}
& c_{0}=972\left(-64 M_{2} M_{4} M_{5}^{2} M_{6}+16 M_{2}^{2} M_{4} M_{5}^{3}+16 M_{2}^{2} M_{4}^{2} M_{5}^{2}+16 M_{2}^{3} M_{5}^{2} M_{6}-8 M_{2}^{4} M_{4} M_{5}^{2}\right. \\
& \left.-16 M_{2} M_{5}^{3} M_{6}-16 M_{4}^{2} M_{5}^{3}-8 M_{4} M_{5}^{4}+64 M_{5}^{2} M_{6}^{2}+3 M_{2}^{2} M_{5}^{4}-3 M_{2}^{4} M_{5}^{3}+M_{2}^{6} M_{5}^{2}-M_{5}^{5}\right) \text {, } \\
& c_{1}=1296\left(3 M_{1} M_{2}^{5} M_{5}^{2}-9 M_{2}^{4} M_{3} M_{5}^{2}+36 M_{1} M_{5}^{3} M_{6}-32 M_{3} M_{4} M_{5}^{3}-64 M_{1} M_{2} M_{5} M_{6}^{2}\right. \\
& -M_{1} M_{2}^{7} M_{5}+4 M_{1} M_{2} M_{4} M_{5}^{3}+128 M_{3} M_{5} M_{6}^{2}+48 M_{2}^{2} M_{3} M_{4} M_{5}^{2}+32 M_{2}^{2} M_{3} M_{4}^{2} M_{5} \\
& +48 M_{1} M_{4} M_{5}^{2} M_{6}-16 M_{2}^{4} M_{3} M_{4} M_{5}-48 M_{2} M_{3} M_{5}^{2} M_{6}+12 M_{1} M_{2}^{2} M_{5}^{2} M_{6} \\
& +32 M_{2}^{3} M_{3} M_{5} M_{6}-12 M_{1} M_{2}^{3} M_{4} M_{5}^{2}+8 M_{1} M_{2}^{5} M_{4} M_{5}-16 M_{1} M_{2}^{4} M_{5} M_{6} \\
& -16 M_{1} M_{2}^{3} M_{4}^{2} M_{5}+64 M_{1} M_{2}^{2} M_{4} M_{5} M_{6}-128 M_{2} M_{3} M_{4} M_{5} M_{6}+M_{1} M_{2} M_{5}^{4} \\
& \left.+12 M_{2}^{2} M_{3} M_{5}^{3}-3 M_{1} M_{2}^{3} M_{5}^{3}-48 M_{3} M_{4}^{2} M_{5}^{2}+2 M_{2}^{6} M_{3} M_{5}-5 M_{3} M_{5}^{4}\right) \text {, } \\
& c_{2}=108\left(256 M_{2}^{2} M_{3}^{2} M_{4}^{2}+2736 M_{2} M_{4}^{2} M_{5}^{2}+252 M_{2} M_{4} M_{5}^{3}-55296 M_{2} M_{4} M_{6}^{2}\right. \\
& +2124 M_{2}^{5} M_{4} M_{5}-128 M_{2}^{4} M_{3}^{2} M_{4}-6192 M_{2}^{3} M_{4}^{2} M_{5}+256 M_{2}^{3} M_{3}^{2} M_{6} \\
& -13824 M_{2}^{4} M_{4} M_{6}-10368 M_{4}^{2} M_{5} M_{6}-768 M_{3}^{2} M_{4} M_{5}^{2}-4608 M_{2}^{3} M_{4}^{3}-864 M_{2}^{7} M_{4} \\
& -225 M_{2}^{7} M_{5}+16 M_{2}^{6} M_{3}^{2}+207 M_{2}^{5} M_{5}^{2}+1024 M_{3}^{2} M_{6}^{2}-27 M_{2} M_{5}^{4}+3456 M_{2}^{5} M_{4}^{2} \\
& +1728 M_{2}^{6} M_{6}-18 M_{1}^{2} M_{5}^{4}-1224 M_{5}^{3} M_{6}+13824 M_{2}^{3} M_{6}^{2}-27 M_{2}^{3} M_{5}^{3} \\
& +4 M_{1}^{2} M_{2}^{8}-160 M_{3}^{2} M_{5}^{3}+1536 M_{1} M_{3} M_{4} M_{5} M_{6}-1920 M_{1}^{2} M_{2} M_{4} M_{5} M_{6} \\
& +192 M_{1} M_{2} M_{3} M_{4} M_{5}^{2}+384 M_{1} M_{2}^{2} M_{3} M_{5} M_{6}+1024 M_{1} M_{2}^{2} M_{3} M_{4} M_{6} \\
& -384 M_{1} M_{2}^{3} M_{3} M_{4} M_{5}+72 M_{2}^{9}+36864 M_{6}^{3}+768 M_{2}^{2} M_{3}^{2} M_{4} M_{5}-768 M_{2} M_{3}^{2} M_{5} M_{6} \\
& +1728 M_{1} M_{3} M_{5}^{2} M_{6}-16 M_{1} M_{2}^{7} M_{3}-256 M_{1} M_{2}^{3} M_{3} M_{4}^{2}-144 M_{1} M_{2}^{3} M_{3} M_{5}^{2} \\
& -1440 M_{1}^{2} M_{2} M_{5}^{2} M_{6}-256 M_{1} M_{2}^{4} M_{3} M_{6}+128 M_{1} M_{2}^{5} M_{3} M_{4}+480 M_{1}^{2} M_{2}^{2} M_{4}^{2} M_{5} \\
& +96 M_{1} M_{2}^{5} M_{3} M_{5}+14400 M_{2}^{2} M_{4} M_{5} M_{6}-1024 M_{1} M_{2} M_{3} M_{6}^{2}+64 M_{1} M_{2} M_{3} M_{5}^{3} \\
& +528 M_{1}^{2} M_{2}^{2} M_{4} M_{5}^{2}+1872 M_{2}^{2} M_{5}^{2} M_{6}-768 M_{3}^{2} M_{4}^{2} M_{5}+64 M_{1}^{2} M_{2}^{4} M_{4}^{2} \\
& -1512 M_{2}^{3} M_{4} M_{5}^{2}-288 M_{1}^{2} M_{4} M_{5}^{3}+256 M_{1}^{2} M_{2}^{2} M_{6}^{2}+27648 M_{2}^{2} M_{4}^{2} M_{6} \\
& +5184 M_{2} M_{4}^{3} M_{5}-144 M_{2}^{4} M_{3}^{2} M_{5}-9216 M_{2} M_{5} M_{6}^{2}-6336 M_{4} M_{5}^{2} M_{6} \\
& +98 M_{1}^{2} M_{2}^{2} M_{5}^{3}-2952 M_{2}^{4} M_{5} M_{6}+6 M_{1}^{2} M_{2}^{6} M_{5}-32 M_{1}^{2} M_{2}^{6} M_{4}+1152 M_{1}^{2} M_{5} M_{6}^{2} \\
& +64 M_{1}^{2} M_{2}^{5} M_{6}-288 M_{1}^{2} M_{4}^{2} M_{5}^{2}-90 M_{1}^{2} M_{2}^{4} M_{5}^{2}+288 M_{1}^{2} M_{2}^{3} M_{5} M_{6}-144 M_{1}^{2} M_{2}^{4} M_{4} M_{5} \\
& \left.-256 M_{1}^{2} M_{2}^{3} M_{4} M_{6}-1024 M_{2} M_{3}^{2} M_{4} M_{6}+288 M_{2}^{2} M_{3}^{2} M_{5}^{2}\right) \text {, } \\
& c_{3}=36\left(-1024 M_{2} M_{3}^{3} M_{6}+3528 M_{1} M_{2}^{6} M_{4}+20736 M_{2} M_{3} M_{4}^{3}-81 M_{1} M_{2}^{4} M_{5}^{2}\right. \\
& -14688 M_{3} M_{5}^{2} M_{6}-324 M_{2}^{3} M_{3} M_{5}^{2}-2048 M_{3}^{3} M_{4} M_{5}+1404 M_{4} M_{5}^{3} M_{1} \\
& -832 M_{1}^{3} M_{2}^{3} M_{4}^{2}-172 M_{1}^{3} M_{2}^{3} M_{5}^{2}-5616 M_{1} M_{4}^{2} M_{5}^{2}+768 M_{2}^{2} M_{3}^{3} M_{5}-640 M_{1}^{3} M_{2}^{4} M_{6}
\end{aligned}
$$




$$
\begin{aligned}
& +82944 M_{1} M_{5} M_{6}^{2}+8496 M_{2}^{5} M_{3} M_{4}+1656 M_{2}^{5} M_{3} M_{5}+352 M_{1}^{3} M_{2}^{5} M_{4} \\
& -41472 M_{3} M_{4}^{2} M_{6}-24768 M_{2}^{3} M_{3} M_{4}^{2}+4320 M_{1}^{3} M_{5}^{2} M_{6}+165888 M_{1} M_{4} M_{6}^{2} \\
& -23040 M_{1} M_{2}^{2} M_{6}^{2}-4464 M_{1} M_{2}^{5} M_{6}-288 M_{1}^{2} M_{3} M_{5}^{3}+24 M_{1}^{2} M_{2}^{6} M_{3} \\
& +31104 M_{1} M_{2}^{2} M_{4}^{3}-18720 M_{1} M_{2}^{4} M_{4}^{2}-15552 M_{1} M_{4}^{3} M_{5}-36864 M_{2} M_{3} M_{6}^{2} \\
& +300 M_{1}^{3} M_{2}^{5} M_{5}-432 M_{2} M_{3} M_{5}^{3}-2304 M_{1}^{3} M_{2} M_{6}^{2}-11808 M_{2}^{4} M_{3} M_{6} \\
& -567 M_{2}^{2} M_{5}^{3} M_{1}+36 M_{1}^{3} M_{2} M_{5}^{3}+4608 M_{1}^{2} M_{3} M_{6}^{2}-576 M_{1} M_{2}^{3} M_{3}^{2} M_{5} \\
& \text { - } 768 M_{1} M_{2}^{3} M_{3}^{2} M_{4}-6372 M_{1} M_{2}^{4} M_{4} M_{5}-1184 M_{1}^{3} M_{2}^{3} M_{4} M_{5}+54144 M_{1} M_{2}^{3} M_{4} M_{6} \\
& +3024 M_{2} M_{3} M_{4} M_{5}^{2}-12096 M_{2}^{3} M_{3} M_{4} M_{5}+6912 M_{1} M_{3}^{2} M_{5} M_{6}-576 M_{1}^{2} M_{2}^{4} M_{3} M_{4} \\
& -720 M_{1}^{2} M_{2}^{4} M_{3} M_{5}+1176 M_{1}^{2} M_{2}^{2} M_{3} M_{5}^{2}+3072 M_{1}^{3} M_{2}^{2} M_{4} M_{6}+3168 M_{1}^{3} M_{2}^{2} M_{5} M_{6} \\
& +1152 M_{1}^{2} M_{2}^{3} M_{3} M_{6}+57600 M_{2}^{2} M_{3} M_{4} M_{6}+21888 M_{2} M_{3} M_{4}^{2} M_{5}+3072 M_{1} M_{3}^{2} M_{4} M_{6} \\
& +11664 M_{1} M_{2} M_{5}^{2} M_{6}+864 M_{1} M_{2}^{2} M_{4} M_{5}^{2}+384 M_{1} M_{2} M_{3}^{2} M_{5}^{2}-145152 M_{1} M_{2} M_{4}^{2} M_{6} \\
& +3456 M_{1}^{3} M_{4} M_{5} M_{6}+19728 M_{1} M_{2}^{2} M_{4}^{2} M_{5}-576 M_{1}^{3} M_{2} M_{4}^{2} M_{5}-50688 M_{3} M_{4} M_{5} M_{6} \\
& +14976 M_{2}^{2} M_{3} M_{5} M_{6}-2304 M_{1}^{2} M_{3} M_{4}^{2} M_{5}-3456 M_{1}^{2} M_{3} M_{4} M_{5}^{2}+1920 M_{1}^{2} M_{2}^{2} M_{3} M_{4}^{2} \\
& +768 M_{1} M_{2}^{2} M_{3}^{2} M_{6}+12384 M_{1} M_{2}^{3} M_{5} M_{6}-198 M_{1} M_{2}^{8}+243 M_{1} M_{5}^{4}-640 M_{3}^{3} M_{5}^{2} \\
& -36 M_{1}^{3} M_{2}^{7}-1024 M_{3}^{3} M_{4}^{2}-900 M_{2}^{7} M_{3}-192 M_{2}^{4} M_{3}^{3}-7680 M_{1}^{2} M_{2} M_{3} M_{4} M_{6} \\
& -11520 M_{1}^{2} M_{2} M_{3} M_{5} M_{6}-54144 M_{1} M_{2} M_{4} M_{5} M_{6}+768 M_{1} M_{2} M_{3}^{2} M_{4} M_{5} \\
& \left.+4224 M_{1}^{2} M_{2}^{2} M_{3} M_{4} M_{5}+192 M_{1} M_{2}^{5} M_{3}^{2}+603 M_{1} M_{2}^{6} M_{5}+1024 M_{2}^{2} M_{3}^{3} M_{4}\right) \text {, } \\
& c_{4}=3\left(-8667 M_{2}^{8}-324 M_{1}^{4} M_{5}^{3}-1492992 M_{5} M_{6}^{2}+20736 M_{1}^{4} M_{6}^{2}-444 M_{1}^{4} M_{2}^{6}\right. \\
& -16236 M_{1}^{2} M_{2}^{7}-2592 M_{4}^{2} M_{5}^{2}-11826 M_{2}^{4} M_{5}^{2}+13248 M_{2}^{5} M_{3}^{2}-186624 M_{4}^{3} M_{5} \\
& +311040 M_{2}^{2} M_{4}^{3}-5120 M_{3}^{4} M_{5}-8192 M_{3}^{4} M_{4}-3981312 M_{4} M_{6}^{2}+331776 M_{2}^{2} M_{6}^{2} \\
& -189216 M_{2}^{4} M_{4}^{2}-10368 M_{2}^{5} M_{6}+17820 M_{2}^{6} M_{5}+71280 M_{2}^{6} M_{4}+3072 M_{2}^{2} M_{3}^{4} \\
& -5504 M_{1}^{3} M_{2}^{3} M_{3} M_{5}-2187 M_{5}^{4}+29376 M_{1}^{4} M_{2}^{2} M_{4} M_{5}-18944 M_{1}^{3} M_{2}^{3} M_{3} M_{4} \\
& -48384 M_{1}^{4} M_{2} M_{4} M_{6}-95040 M_{1}^{4} M_{2} M_{5} M_{6}+198144 M_{1} M_{2}^{3} M_{3} M_{6}-2592 M_{1} M_{2}^{4} M_{3} M_{5} \\
& \text { - 101952 } M_{1} M_{2}^{4} M_{3} M_{4}-20736 M_{1}^{2} M_{4} M_{5} M_{6}-92160 M_{1}^{2} M_{2} M_{3}^{2} M_{6}-55296 M_{1}^{2} M_{3}^{2} M_{4} M_{5} \\
& +33792 M_{1}^{2} M_{2}^{2} M_{3}^{2} M_{4}+18816 M_{1}^{2} M_{2}^{2} M_{3}^{2} M_{5}+219456 M_{1}^{2} M_{2} M_{4}^{2} M_{5}-112320 M_{1}^{2} M_{2}^{2} M_{5} M_{6} \\
& +315648 M_{1} M_{2}^{2} M_{3} M_{4}^{2}+4096 M_{1} M_{2} M_{3}^{3} M_{4}+1728 M_{1}^{3} M_{2} M_{3} M_{5}^{2}+138240 M_{1}^{3} M_{3} M_{5} M_{6} \\
& +4096 M_{1} M_{2} M_{3}^{3} M_{5}-9216 M_{1}^{3} M_{2} M_{3} M_{4}^{2}+663552 M_{2} M_{4} M_{5} M_{6}+55296 M_{1}^{3} M_{3} M_{4} M_{6} \\
& +1052928 M_{1}^{2} M_{2}^{2} M_{4} M_{6}-27216 M_{2}^{2} M_{5}^{2} M_{1} M_{3}+16848 M_{4} M_{5}^{2} M_{1}^{2} M_{2}+67392 M_{4} M_{5}^{2} M_{1} M_{3} \\
& +48384 M_{2} M_{3}^{2} M_{4} M_{5}-179712 M_{1} M_{3} M_{4}^{2} M_{5}-95904 M_{1}^{2} M_{2}^{3} M_{4} M_{5}+50688 M_{1}^{3} M_{2}^{2} M_{3} M_{6} \\
& -5760 M_{1}^{2} M_{2}^{4} M_{3}^{2}+15552 M_{1} M_{5}^{3} M_{3}+120528 M_{1}^{2} M_{2}^{5} M_{4}-186624 M_{1}^{2} M_{2} M_{4}^{3} \\
& -1492992 M_{1}^{2} M_{2} M_{6}^{2}-175680 M_{1}^{2} M_{2}^{3} M_{4}^{2}-6912 M_{1}^{2} M_{3}^{2} M_{5}^{2}-297504 M_{1}^{2} M_{2}^{4} M_{6} \\
& -12960 M_{1}^{4} M_{4} M_{5}^{2}+53136 M_{2}^{2} M_{5}^{2} M_{4}-324 M_{1}^{2} M_{2} M_{5}^{3}-1568 M_{1}^{4} M_{2}^{4} M_{4} \\
& -5708 M_{1}^{4} M_{2}^{4} M_{5}-1728 M_{1}^{4} M_{2}^{3} M_{6}-5184 M_{1}^{4} M_{4}^{2} M_{5}-10368 M_{2} M_{3}^{2} M_{5}^{2} \\
& -209952 M_{6} M_{1}^{2} M_{5}^{2}+4800 M_{1}^{3} M_{2}^{5} M_{3}-82944 M_{2}^{3} M_{5} M_{6}+4428 M_{1}^{4} M_{2}^{2} M_{5}^{2} \\
& +1327104 M_{1} M_{3} M_{6}^{2}-155520 M_{2} M_{5}^{2} M_{6}-96768 M_{2}^{3} M_{3}^{2} M_{4}-5184 M_{2}^{3} M_{3}^{2} M_{5}
\end{aligned}
$$


$+2488320 M_{2} M_{4}^{2} M_{6}-405504 M_{3}^{2} M_{4} M_{6}-235008 M_{3}^{2} M_{5} M_{6}+119808 M_{2}^{2} M_{3}^{2} M_{6}$

$-112752 M_{2}^{4} M_{4} M_{5}-248832 M_{1} M_{3} M_{4}^{3}+175104 M_{2} M_{3}^{2} M_{4}^{2}+212544 M_{2}^{2} M_{4}^{2} M_{5}$

$-18432 M_{1}^{2} M_{3}^{2} M_{4}^{2}+622080 M_{1}^{2} M_{4}^{2} M_{6}-3072 M_{1} M_{2}^{3} M_{3}^{3}+9648 M_{1} M_{2}^{6} M_{3}$

$+36864 M_{1} M_{3}^{3} M_{6}+14400 M_{1}^{4} M_{2}^{2} M_{4}^{2}+2268 M_{2}^{3} M_{5}^{2} M_{1}^{2}+11988 M_{1}^{2} M_{2}^{5} M_{5}$

$+373248 M_{1} M_{2} M_{3} M_{5} M_{6}+27648 M_{1} M_{2}^{2} M_{3} M_{4} M_{5}-866304 M_{1} M_{2} M_{3} M_{4} M_{6}$

$\left.-580608 M_{2}^{3} M_{4} M_{6}-11664 M_{4} M_{5}^{3}-559872 M_{4}^{4}+4860 M_{2}^{2} M_{5}^{3}\right)$,

$c_{5}=4\left(-1024 M_{3}^{5}+632448 M_{1} M_{2}^{3} M_{4}^{2}+11988 M_{1}^{2} M_{2}^{5} M_{3}-23652 M_{2}^{4} M_{3} M_{5}\right.$

$-6912 M_{2} M_{3}^{3} M_{5}+16128 M_{2} M_{3}^{3} M_{4}-159408 M_{1}^{3} M_{4}^{2} M_{5}-82944 M_{2}^{3} M_{3} M_{6}$

$+69120 M_{1}^{3} M_{3}^{2} M_{6}-18432 M_{1}^{2} M_{3}^{3} M_{4}-4608 M_{1}^{2} M_{3}^{3} M_{5}+6272 M_{1}^{2} M_{2}^{2} M_{3}^{3}$

$+1024 M_{1} M_{2} M_{3}^{4}-5184 M_{1}^{5} M_{2} M_{4}^{2}-1296 M_{1} M_{2}^{4} M_{3}^{2}+186624 M_{1} M_{2} M_{4}^{3}$

$-89856 M_{1} M_{3}^{2} M_{4}^{2}+1029024 M_{1} M_{2}^{4} M_{6}-13365 M_{2}^{2} M_{5}^{2} M_{1}^{3}+19440 M_{2}^{3} M_{5}^{2} M_{1}$

$-34992 M_{4} M_{5}^{2} M_{3}+14580 M_{2}^{2} M_{5}^{2} M_{3}-5184 M_{1}^{4} M_{3} M_{4}^{2}+15552 M_{1}^{5} M_{4} M_{6}$

$+42768 M_{1}^{5} M_{5} M_{6}-185940 M_{1}^{3} M_{2}^{4} M_{4}-2752 M_{1}^{3} M_{2}^{3} M_{3}^{2}-5184 M_{4}^{2} M_{5} M_{3}$

$+349920 M_{6} M_{1} M_{5}^{2}+5225472 M_{1} M_{2} M_{6}^{2}+373248 M_{1} M_{4}^{2} M_{6}+576 M_{1}^{5} M_{2}^{3} M_{5}$

$+26352 M_{1}^{5} M_{2}^{2} M_{6}-8496 M_{1}^{5} M_{2}^{3} M_{4}-5708 M_{1}^{4} M_{2}^{4} M_{3}+384768 M_{1}^{3} M_{2}^{3} M_{6}$

$+375408 M_{1}^{3} M_{2}^{2} M_{4}^{2}+39852 M_{1}^{3} M_{5}^{2} M_{4}-972 M_{1}^{4} M_{5}^{2} M_{3}+23328 M_{1} M_{5}^{2} M_{3}^{2}$

$-59616 M_{1} M_{2}^{5} M_{5}-112752 M_{2}^{4} M_{3} M_{4}+2241 M_{1}^{3} M_{5} M_{2}^{4}+40176 M_{1} M_{2}^{7}$

$+212544 M_{2}^{2} M_{3} M_{4}^{2}+17820 M_{2}^{6} M_{3}-419904 M_{6} M_{1}^{2} M_{3} M_{5}+4536 M_{1}^{2} M_{2}^{3} M_{3} M_{5}$

$-972 M_{1}^{2} M_{2} M_{5}^{2} M_{3}-279936 M_{6} M_{2}^{2} M_{1} M_{5}+248832 M_{6} M_{1}^{3} M_{2} M_{5}+13824 M_{1} M_{2}^{2} M_{3}^{2} M_{4}$

$-330480 M_{1} M_{2}^{5} M_{4}-186624 M_{3} M_{4}^{3}-1492992 M_{3} M_{6}^{2}-78336 M_{3}^{3} M_{6}-15552 M_{1}^{3} M_{4}^{3}$

$+22977 M_{1}^{3} M_{2}^{6}+1119744 M_{1}^{3} M_{6}^{2}+243 M_{1}^{3} M_{5}^{3}-1728 M_{2}^{3} M_{3}^{3}+2240 M_{1}^{5} M_{2}^{5}$

$-8748 M_{5}^{3} M_{3}+33696 M_{1}^{2} M_{2} M_{3} M_{4} M_{5}+29376 M_{1}^{4} M_{2}^{2} M_{3} M_{4}+8856 M_{1}^{4} M_{2}^{2} M_{3} M_{5}$

- $95040 M_{1}^{4} M_{2} M_{3} M_{6}-25920 M_{1}^{4} M_{3} M_{4} M_{5}-20736 M_{1}^{2} M_{3} M_{4} M_{6}$

$+219456 M_{1}^{2} M_{2} M_{3} M_{4}^{2}-311040 M_{2} M_{3} M_{5} M_{6}-6480 M_{1}^{5} M_{2} M_{4} M_{5}$

$+663552 M_{2} M_{3} M_{4} M_{6}-95904 M_{1}^{2} M_{2}^{3} M_{3} M_{4}-112320 M_{1}^{2} M_{2}^{2} M_{3} M_{6}$

$+1728 M_{1}^{3} M_{2} M_{3}^{2} M_{5}-27216 M_{1} M_{2}^{2} M_{3}^{2} M_{5}+106272 M_{2}^{2} M_{4} M_{5} M_{3}$

$+386208 M_{2}^{3} M_{5} M_{4} M_{1}-97200 M_{4} M_{5}^{2} M_{1} M_{2}+67392 M_{1} M_{3}^{2} M_{4} M_{5}$

$+186624 M_{1} M_{2} M_{3}^{2} M_{6}-4375296 M_{1} M_{2}^{2} M_{4} M_{6}-1451520 M_{1}^{3} M_{2} M_{4} M_{6}$

$\left.+746496 M_{6} M_{1} M_{4} M_{5}-611712 M_{1} M_{2} M_{4}^{2} M_{5}+18792 M_{1}^{3} M_{2}^{2} M_{4} M_{5}\right)$,

$c_{6}=48\left(64 M_{1}^{3} M_{2} M_{3}^{3}+1044 M_{1}^{6} M_{2}^{2} M_{4}+44064 M_{1}^{4} M_{4} M_{6}+252 M_{2}^{3} M_{3}^{2} M_{1}^{2}\right.$

$-1008 M_{2}^{2} M_{3}^{3} M_{1}+6924 M_{1}^{4} M_{4} M_{2}^{3}-108 M_{1}^{4} M_{5} M_{3}^{2}+249 M_{1}^{3} M_{2}^{4} M_{3}$

$+1728 M_{1} M_{5} M_{3}^{3}+81 M_{1}^{3} M_{5}^{2} M_{3}-972 M_{1}^{4} M_{2}^{3} M_{5}+1620 M_{2}^{2} M_{5} M_{3}^{2}$

$+810 M_{2}^{2} M_{5}^{2} M_{1}^{2}+2496 M_{1} M_{3}^{3} M_{4}+3996 M_{1}^{2} M_{2}^{4} M_{5}+5904 M_{2}^{2} M_{3}^{2} M_{4}$

$-6624 M_{1} M_{2}^{5} M_{3}-84528 M_{1}^{2} M_{2}^{2} M_{4}^{2}-23328 M_{6} M_{1}^{2} M_{3}^{2}+108 M_{1}^{6} M_{5} M_{2}^{2}$

$-17280 M_{2} M_{3}^{2} M_{6}-3888 M_{4} M_{5} M_{3}^{2}-324 M_{1}^{6} M_{4} M_{5}-17712 M_{1}^{3} M_{3} M_{4}^{2}$ 


$$
\begin{aligned}
& +64 M_{1}^{5} M_{2}^{3} M_{3}-3024 M_{1}^{6} M_{2} M_{6}-15120 M_{6} M_{1}^{4} M_{2}^{2}-15552 M_{6} M_{1}^{4} M_{5} \\
& -142128 M_{6} M_{2}^{3} M_{1}^{2}+285120 M_{2}^{2} M_{4} M_{6}+19440 M_{6} M_{2}^{2} M_{5}-46656 M_{6} M_{4} M_{5} \\
& +25920 M_{4}^{2} M_{5} M_{2}+45360 M_{1}^{2} M_{4}^{2} M_{5}-1440 M_{1}^{4} M_{3}^{2} M_{4}-12528 M_{1}^{4} M_{2} M_{4}^{2} \\
& +492 M_{1}^{4} M_{2}^{2} M_{3}^{2}+4752 M_{1}^{5} M_{3} M_{6}+5832 M_{2} M_{4} M_{5}^{2}-1296 M_{2}^{7}+11016 M_{2}^{5} M_{4} \\
& -1314 M_{2}^{4} M_{3}^{2}-15552 M_{2}^{3} M_{4}^{2}+42912 M_{1} M_{2}^{3} M_{3} M_{4}-16848 M_{2}^{3} M_{5} M_{4} \\
& -1296 M_{2}^{3} M_{5}^{2}-2430 M_{4} M_{5}^{2} M_{1}^{2}-31104 M_{4}^{2} M_{6}-17496 M_{6} M_{5}^{2}-373248 M_{2} M_{6}^{2} \\
& -236 M_{1}^{6} M_{2}^{4}-128 M_{1}^{2} M_{3}^{4}-38880 M_{1}^{2} M_{4}^{3}-900 M_{2}^{5} M_{1}^{4}+2592 M_{2}^{5} M_{5} \\
& -1458 M_{5}^{2} M_{3}^{2}-653184 M_{1}^{2} M_{6}^{2}-64152 M_{2}^{4} M_{6}-192 M_{2} M_{3}^{4} \\
& -25812 M_{2}^{2} M_{4} M_{5} M_{1}^{2}+4320 M_{2}^{3} M_{5} M_{3} M_{1}-108 M_{1}^{2} M_{5} M_{3}^{2} M_{2}-67968 M_{1} M_{2} M_{4}^{2} M_{3} \\
& +2088 M_{1}^{3} M_{2}^{2} M_{4} M_{3}+77760 M_{6} M_{1} M_{5} M_{3}-11664 M_{6} M_{1}^{2} M_{5} M_{2} \\
& -720 M_{1}^{5} M_{2} M_{3} M_{4}-31104 M_{6} M_{2}^{2} M_{1} M_{3}-2970 M_{1}^{3} M_{2}^{2} M_{5} M_{3}+27648 M_{6} M_{1}^{3} M_{2} M_{3} \\
& +575424 M_{6} M_{1}^{2} M_{4} M_{2}+82944 M_{6} M_{1} M_{4} M_{3}+1872 M_{1}^{2} M_{3}^{2} M_{4} M_{2} \\
& +4644 M_{1}^{4} M_{5} M_{4} M_{2}+8856 M_{1}^{3} M_{4} M_{5} M_{3}-21600 M_{1} M_{3} M_{4} M_{5} M_{2}-288 M_{3}^{2} M_{4}^{2} \\
& \left.-31104 M_{2} M_{4}^{3}-5958 M_{1}^{2} M_{2}^{6}+47538 M_{1}^{2} M_{2}^{4} M_{4}\right) \text {, } \\
& c_{7}=576\left(-288 M_{2}^{3} M_{3} M_{5}+1728 M_{1} M_{2}^{2} M_{4} M_{5}+1296 M_{2} M_{3} M_{4} M_{5}+60 M_{2}^{2} M_{3}^{3}\right. \\
& -1200 M_{1} M_{2} M_{3}^{2} M_{4}-288 M_{1} M_{2}^{4} M_{5}-2868 M_{1}^{2} M_{2}^{2} M_{3} M_{4}+48 M_{1} M_{3}^{4}+8 M_{1}^{7} M_{2}^{3} \\
& -108 M_{3}^{3} M_{5}+5 M_{2}^{4} M_{1}^{5}+108 M_{1}^{7} M_{6}-108 M_{1}^{5} M_{4}^{2}+93312 M_{1} M_{6}^{2}-4 M_{1}^{4} M_{3}^{3} \\
& +240 M_{1} M_{2}^{3} M_{3}^{2}-2304 M_{1} M_{2}^{4} M_{4}-2028 M_{1}^{3} M_{2}^{3} M_{4}-1872 M_{2}^{3} M_{3} M_{4}+444 M_{1}^{2} M_{2}^{4} M_{3} \\
& +2880 M_{2} M_{3} M_{4}^{2}+2016 M_{1} M_{2}^{2} M_{4}^{2}+3744 M_{1}^{3} M_{2} M_{4}^{2}+5040 M_{1}^{2} M_{3} M_{4}^{2} \\
& -3 M_{1}^{5} M_{4} M_{2}^{2}+972 M_{6} M_{1}^{3} M_{5}-11664 M_{6} M_{1}^{3} M_{4}-108 M_{1}^{4} M_{2}^{3} M_{3}+72 M_{1}^{3} M_{2}^{3} M_{5} \\
& -9 M_{1}^{5} M_{2}^{2} M_{5}+12 M_{1}^{6} M_{2}^{2} M_{3}-4 M_{1}^{2} M_{2} M_{3}^{3}+492 M_{1}^{3} M_{3}^{2} M_{4}+27 M_{1}^{5} M_{5} M_{4} \\
& -36 M_{1}^{7} M_{2} M_{4}-36 M_{1}^{6} M_{3} M_{4}-1728 M_{6} M_{1}^{4} M_{3}+3780 M_{6} M_{1}^{3} M_{2}^{2}+216 M_{6} M_{1}^{5} M_{2} \\
& +15552 M_{6} M_{2}^{3} M_{1}-3888 M_{3} M_{6} M_{5}+4320 M_{6} M_{1} M_{3}^{2}-2592 M_{1} M_{4}^{2} M_{5}-5184 M_{6} M_{4} M_{3} \\
& +2160 M_{6} M_{2}^{2} M_{3}-165 M_{2}^{2} M_{3}^{2} M_{1}^{3}+9 M_{1}^{3} M_{5} M_{3}^{2}-144 M_{3}^{3} M_{4}-324 M_{1}^{3} M_{4} M_{5} M_{2} \\
& +180 M_{2}^{2} M_{3} M_{5} M_{1}^{2}+516 M_{1}^{4} M_{2} M_{4} M_{3}-67392 M_{6} M_{1} M_{4} M_{2}-1296 M_{6} M_{1}^{2} M_{3} M_{2} \\
& \left.-540 M_{1}^{2} M_{4} M_{5} M_{3}+288 M_{1} M_{2}^{6}+264 M_{1}^{3} M_{2}^{5}+288 M_{2}^{5} M_{3}+10368 M_{1} M_{4}^{3}\right) \text {, } \\
& c_{8}=2304\left(-96 M_{1} M_{2}^{4} M_{3}-48 M_{2}^{3} M_{3}^{2}-1728 M_{4}^{3}+576 M_{1} M_{2}^{2} M_{3} M_{4}-648 M_{3}^{2} M_{6}\right. \\
& +M_{1}^{3} M_{3}^{3}-27 M_{1}^{6} M_{6}-2 M_{1}^{6} M_{2}^{3}-9 M_{3}^{4}+432 M_{2}^{2} M_{4}^{2}+24 M_{1}^{3} M_{2}^{3} M_{3}-90 M_{1}^{2} M_{3}^{2} M_{4} \\
& +30 M_{1}^{2} M_{2}^{2} M_{3}^{2}-648 M_{1}^{2} M_{2} M_{4}^{2}-11664 M_{6}^{2}-18 M_{1}^{4} M_{2}^{2} M_{4}-3 M_{1}^{5} M_{2}^{2} M_{3} \\
& +324 M_{1}^{3} M_{3} M_{6}+7776 M_{2} M_{4} M_{6}+9 M_{1}^{6} M_{2} M_{4}+9 M_{1}^{5} M_{3} M_{4}-648 M_{1}^{2} M_{2}^{2} M_{6} \\
& -864 M_{1} M_{3} M_{4}^{2}-108 M_{1}^{3} M_{2} M_{3} M_{4}-1728 M_{6} M_{2}^{3}+27 M_{1}^{4} M_{4}^{2}+3 M_{1}^{4} M_{2}^{4} \\
& \left.+1944 M_{6} M_{1}^{2} M_{4}+360 M_{1}^{2} M_{2}^{3} M_{4}+216 M_{2} M_{3}^{2} M_{4}-48 M_{1}^{2} M_{2}^{5}\right) \text {. }
\end{aligned}
$$




\section{References}

[1] W. Dür, G. Vidal, and J. I. Cirac. Three qubits can be entangled in two inequivalent ways. Physical Review A 62, 062314, 2000.

[2] L. Chen and Y.-X. Chen. Range criterion and classification of true entanglement in a $2 \times M \times N$ system. Physical Review A 73, 052310, 2006.

[3] L. Chen, E. Chitambar, R. Duan, Z. Ji, and A. Winter. Tensor rank and stochastic entanglement catalysis for multipartite pure states. Physical Review Letters 105, 200501, 2010.

[4] L. Amico, R. Fazio, A. Osterloh, and V. Vedral. Entanglement in many-body systems. Reviews of Modern Physics 80, 517-576, 2008.

[5] J. Schliemann, D. Loss, and A. H. MacDonald. Double-occupancy errors, adiabaticity, and entanglement of spin qubits in quantum dots. Physical Review B 63, 085311, 2001.

[6] J. Schliemann, J. I. Cirac, M. Kuś, M. Lewenstein, Lewenstein, and D. Loss. Quantum correlations in two-fermion systems. Physical Review A 64, 022303, 2001.

[7] Y. S. Li, B. Zeng, X. S. Liu, and G. L. Long. Entanglement in a two-identical-particle system. Physical Review A 64, 054302, 2001.

[8] P. Paškauskas and L. You. Quantum correlations in two-boson wave functions. Physical Review A 64, 042310, 2001.

[9] K. Eckert, J. Schliemann, D. Bruß, and M. Lewenstein. Quantum correlations in systems of indistinguishable particles. Annals of Physics 299, 88-127, 2002.

[10] L. Chen, J. Chen, D. Ž. Đoković, and B. Zeng. Universal subspaces for local unitary groups of fermionic systems. arXiv:1301.3421v1 [quant-ph], 15 Jan 2013.

[11] A. Acín, A. Andrianov, E. Jané, and R. Tarrach. Three-qubit pure-state canonical forms. Journal of Physics A: Mathematical and General 34, 6725, 2001.

[12] F. Verstraete, J. Dehaene, B. De Moor, and H. Verschelde. Four qubits can be entangled in nine different ways. Physical Review A 65, 052112, 2002.

[13] O. Chterental and D. Ž. Đoković. Normal forms and tensor ranks of pure states of four qubits. In G. D. Ling, editor, Linear Algebra Research Advances, chapter 4, pages 133-167. Nova Science Publishers, 2007.

[14] P. Mathonet, S. Krins, M. Godefroid, L. Lamata, E. Solano, and T. Bastin. Entanglement equivalence of $N$-qubit symmetric states. Physical Review A 81, 052315, 2010.

[15] M. Walter, B. Doran, D. Gross, and M. Christandl. Entanglement polytopes. arXiv:1208.0365 [quant-ph], 2012. 
[16] A. Sawicki, M. Oszmaniec, and M. Kuś. Convexity of momentum map, Morse index, and quantum entanglement. arXiv:1208.0556 [quant-ph], 2012.

[17] A. Sawicki, M. Oszmaniec, and M. Kuś. Critical sets of the total variance of state detect all SLOCC entanglement classes. arXiv:1208.0557 [quant-ph], 2012.

[18] P. Lévay and P. Vrana. Three fermions with six single-particle states can be entangled in two inequivalent ways. Physical Review A 78, 022329, 2008.

[19] J. A. Schouten. Klassifizierung der alternierenden Gröszen dritten Grades in 7 Dimensionen. Rendiconti del Circolo Matematico di Palermo 55, 137-156, 1931.

[20] R. Ehrenborg. Canonical forms of two by two by two matrices. Journal of Algebra 213, 195-224, 1999.

[21] A. J. Coleman. Structure of fermion density matrices. Reviews of Modern Physics 35, 668-686, 1963.

[22] A. A. Klyachko. Quantum marginal problem and N-representability. Journal of Physics: Conference Series 36, 2006.

[23] R. E. Borland and K. Dennis. The conditions on the one-matrix for three-body fermion wavefunctions with one-rank equal to six. Journal of Physics B 5, 7-15, 1972.

[24] M. B. Ruskai. Connecting $N$-representability to Weyl's problem: the one-particle density matrix for $N=3$ and $R=6$. Journal of Physics A: Mathematical and Theoretical 40, F961-F967, 2007.

[25] A. A. Klyachko. The Pauli exclusion principle and beyond. arXiv:0904.2009 [quant-ph], 2009.

[26] A. Shimony. Degree of entanglement. Annals of the New York Academy of Science 755, 675-679, 1995.

[27] T.-C. Wei and P. M. Goldbart. Geometric measure of entanglement and applications to bipartite and multipartite quantum states. Physical Review A 68, 042307, 2003.

[28] D. Gross, S. T. Flammia, and J. Eisert. Most quantum states are too entangled to be useful as computational resources. Physical Review Letters 102, 190501, 2009.

[29] A. W. Harrow and A. Montanaro. An efficient test for product states, with applications to quantum Merlin-Arthur games. In Proceedings 51st Annual Symposium on Foundations of Computer Science (FOCS 2010), pages 633-642, 2010. arXiv:1001.0017v6 [quant-ph].

[30] A. Montanaro. Weak multiplicativity for random quantum channels. Communications in Mathematical Physics 319, 535-555, 2013. 
[31] L. Chen, A. Xu, and H. Zhu. Computation of the geometric measure of entanglement for pure multiqubit states. Physical Review A 82, 032301, 2010.

[32] M. Aulbach, D. Markham, and M. Murao. The maximally entangled symmetric state in terms of the geometric measure. New Journal of Physics 12, 073025, 2010.

[33] M. Hayashi, D. Markham, M. Murao, M. Owari, and S. Virmani. Entanglement of multiparty-stabilizer, symmetric, and antisymmetric states. Physical Review A 77, 012104, 2008.

[34] H. Zhu, L. Chen, and M. Hayashi. Additivity and non-additivity of multipartite entanglement measures. New Journal of Physics 12, 083002, 2010. arXiv:1002.2511 [quantph].

[35] T.-C. Wei, M. Ericsson, P. M. Goldbart, and W. J. Munro. Connections between relative entropy of entanglement and geometric measure of entanglement. Quantum Information E6 Computation 4, 252, 2004.

[36] L. Chen, H. Zhu, and T.-C. Wei. Connections of geometric measure of entanglement of pure symmetric states to quantum state estimation. Physical Review A 83, 012305, 2010.

[37] G. W. Schwarz. Representations of simple Lie groups with regular rings of invariants. Inventiones mathematicae 59, 167-191, 1978.

[38] T. Kimura. Introduction to Prehomogeneous Vector Spaces. American Mathematical Society, Providence, RI, 2003.

[39] A. L. Onishchik and E. B. Vinberg. Lie Groups and Algebraic Groups. Springer, Berlin, 1990.

[40] G. Bredon. Introduction to Compact Transformation Groups. Academic Press, New York, 1972.

[41] Maple 15. Maplesoft, a division of Waterloo Maple Inc., Waterloo, Ontario.

[42] W. Bosma, J. J. Cannon, and C. Playoust. The Magma Algebra System I: The User Language. Journal of Symbolic Computation 24, 235-265, 1997. 